
Risk: Characterizing Utility Functions"

Christian GOLLIER and Miles S. KIMBALL 


\title{
Toward a Systematic Approach to the Economic Effects of Risk: Characterizing Utility Functions
}

\author{
Christian GOLLIER \\ Toulouse School of Economics \\ Miles S. KIMBALL \\ University of Colorado Boulder
}

March 27, 2018

\begin{abstract}
The Diffidence Theorem, together with complementary tools, can aid in illuminating a broad set of questions about how to mathematically characterize the set of utility functions with specified economic properties. This paper establishes the technique and illustrates its application to many questions, old and new. For example, among many other older and other technically more difficult results, it is shown that (1) several implications of globally greater risk aversion depend on distinct mathematical properties when the initial wealth level is known, (2) whether opening up a new asset market increases or decreases saving depends on whether the reciprocal of marginal utility is concave or convex, and (3) whether opening up a new asset market raises or lowers risk aversion towards small independent risks depends on whether absolute risk aversion is convex or concave.
\end{abstract}

Acknowledgement: We would also like to acknowledge funding from the chair SCOR and FDIR at TSE. 


\section{Introduction}

By implying linearity of preferences in probabilities, von Neumann-Morgenstern expected utility theory provides a great deal of common structure for applied problems. Yet the literature on the economics of risk has often approached each applied problem or small class of applied problems in what look like very different ways. In this paper, we endeavor to make a bare beginning to systematizing approaches to applied problems in the economics of risk. In particular, we study a systematic technique for approaching the mathematical characterization of sets of utility functions with particular economic properties. That is, the objective here is to build a path between economic properties of preferences and mathematical properties of utility functions. In a companion paper, "New Methods in the Classical Economics of Uncertainty: Comparing Risks" (Gollier and Kimball, 2018), we address the mathematical characterization of risks that have particular economic properties in relation to specified sets of utility functions.

A spur for the development of more systematic techniques in the economics of risk is the burgeoning and technically difficult literature on background risks. Since Doherty and Schlesinger (1983), Ross (1981), Kihlstrom, Romer and Williams (1981) and Pratt and Zeckhauser (1987), our understanding of the behavior of agents facing more than one source of risk has made a great deal of progress. A common feature of analyses of the interaction between independent risks is the existence of paradoxes that can be solved by putting additional restrictions on the utility function. For example, Kimball (1993), Eeckhoudt, Gollier and Schlesinger (1996), and Gollier and Pratt (1996) showed conditions under which adding a nonmarketable zero-mean risk to wealth can induce a risk-averse agent to purchase more of another independent risky asset. Eeckhoudt and Schlesinger (2006) pushed this literature further.

Difficult background risk problems and more basic problems in the economics of risk often share elements with the same technical structure. More than twenty years ago, we wrote an early version of this paper (Gollier and Kimball, 1994) in which we provided a simple method for solving these problems. The centerpiece of this method is the "Diffidence Theorem." This theorem has since been used by various authors to solve some of these complex problems in a simple way, as we show in examples throughout this paper.

In addition to illustrating a technique of wide applicability, reframing many existing results in useful ways, and establishing many minor new results, we establish at least three important substantive results not in the 
previous literature. First, we clarify the ways in which globally greater risk aversion can be overkill for an applied problem. If something is known about initial starting wealth, the much weaker condition of centrally greater risk aversion or the even weaker condition of centrally greater diffidence will often be enough. This leads to a much deeper understanding of Arrow-Pratt theory. The mathematics is so basic, it is often tucked, in some form, somewhere into proofs in previous papers, but has not been explored fully in its own right. Second, we give a mathematical characterization of the set of utility functions for which opening up a new asset market would lead to less saving, and the antiphonal set of utility functions for which opening up a new asset market would lead to more saving. Third, we characterize the set of utility functions for which opening up a new asset market would make an agent more risk averse toward a small risk independent of the risk in the new asset market.

Let us turn now to the Diffidence Theorem itself. In many instances, the comparative statics problem involving risk can be written in the following form: Under what conditions on functions $f_{1}$ and $f_{2}$ can we guarantee that

$$
\forall \tilde{x}: \quad E f_{1}(\tilde{x}) \leq E f_{1}\left(x_{0}\right) \Longrightarrow E f_{2}(\tilde{x}) \leq E f_{2}\left(x_{0}\right),
$$

for some starting wealth $w$. Let us consider a very simple illustration of problem (1). There are several ways to define the concept of "greater risk aversion". For example, one can stipulate the definition that an agent with utility function $u_{2}$ is more risk-averse than another with utility function $u_{1}$ if and only if any risk $\tilde{x}$ that is rejected by the latter is also rejected by the former, independent of the sure common wealth level $w$ of the two agents. This is an application of problem (1) with $f_{i}(x)=u_{i}(w+x)$ For each initial wealth level, this problem is equivalent to the following property:

$$
\forall \tilde{x}: \quad E u_{1}(w+\tilde{x}) \leq u_{1}\left(x_{0}\right) \Longrightarrow E u_{2}(w+\tilde{x}) \leq u_{2}\left(x_{0}\right),
$$

The concepts of risk aversion, prudence, temperance, decreasing absolute risk aversion, decreasing absolute prudence, proper risk aversion(Pratt and Zeckhauser, 1987), risk vulnerability (Gollier and Pratt, 1996; Gollier 2001), and standard risk aversion (Kimball, 1993) can also be defined by using definition (1) for some specific pair of functions $\left(f_{1}, f_{2}\right)$, or by using its bivariate extension that we also present in this paper. In addition, we consider new concepts having the same structure as in (1). For example, under what condition does an increase in nonmarketable background risk raise the equilibrium risk free rate in the economy? Or, under what condition does opening up a new asset market raise current consumption? And does opening 
a market for a new asset reduce the demand for another independent risky asset? All these questions, and others, are solved in this paper by using the Diffidence Theorem.

The Diffidence Theorem is a consequence of the linearity of expected utility with respect to probabilities. This linearity implies that Condition (1) holds for all random variables if and only if it holds for all binary random variables. This property singularly simplifies the structure of the problem, and the Diffidence Theorem can be seen as an application of that result.

Property (1) is related to the condition that $f_{2}$ is more concave that $f_{1}$ in the sense of Arrow-Pratt. This is an obvious consequence of Jensen's inequality, a ubiquitous tool in decision theory under uncertainty. However, the "more concave" condition is often too restrictive, and the Diffidence Theorem provides the right necessary and sufficient condition for property (1). For example, when considering problem (2) for a given wealth level $w$, it is easy to show that there exist pairs of functions $\left(u_{1}, u_{2}\right)$ for which $u_{2}$ is not more risk-averse than $u_{1}$ in the sense of Arrow-Pratt, but still $u_{2}$ rejects all lotteries that $u_{1}$ rejects. By using the Diffidence Theorem, we easily characterize the notion of "centrally greater diffidence," which is the weakest condition on $\left(u_{1}, u_{2}\right)$ that guarantees $(2)$, and lends the Diffidence Theorem its name because it is the most straightforward application of the theorem. Of course, because this property must hold for small risks, a necessary condition for centrally greater diffidence is that $-u_{2}^{\prime \prime}\left(x_{0}\right) / u_{2}^{\prime}\left(x_{0}\right)$ be larger than $-u^{\prime \prime}\left(x_{0}\right) / u_{1}^{\prime}\left(x_{0}\right)$, but this condition need not hold at other wealth levels.

One of the beauties of the celebrated paper by Pratt (1964) is to show that a condition "in the small" - that is, for small risks - if required to hold at all wealth levels, is sufficient "in the large", that is, for any risk. For example, if individual 2 dislikes all small risks that individual 1 dislikes, and if this is true at all common wealth levels, then it is known that individual 2 also dislikes any risk that individual 1 dislikes. Such a result is not true in general under the structure (1), except in some special cases that we identify in this paper.

In Section 2, we prove the Diffidence Theorem and its Corollary. We illustrate the use of the Diffidence Theorem by presenting many applications in Section 3. Section 4 is devoted to the bivariate Diffidence Theorem and its applications. Other extensions are considered in Section 5. Section 6 concludes. 


\section{The Diffidence Theorem}

In this section, we characterize the set of real-valued functions $f_{2}$ that satisfy condition (1) for a given real-valued function $f_{1}$ and a given value of starting wealth $w{ }^{1}$ The proof of the Diffidence Theorem relies on the following Lemma. $^{2}$

Lemma 1 Condition (1) is satisfied for any distribution of $\tilde{x}$ if and only if it is satisfied (a) for all one-point distributions, and (b) for all two-point distributions that satisfy condition $E f_{1}(\tilde{x}) \leq E f_{1}\left(x_{0}\right)$ as an equality.

Proof: Necessity is obvious. To see sufficiency, note that Condition (1) is equivalent to

$$
\begin{aligned}
\max _{\tilde{x}} & E f_{2}(\tilde{x})-E f_{2}\left(x_{0}\right) \leq 0 \\
\text { s.t. } & E f_{1}(\tilde{x})-E f_{1}\left(x_{0}\right) \leq 0 .
\end{aligned}
$$

Both the objective function and the constraint of this problem are linear in probabilities. Thus it is a linear programming problem on the unit simplex. If the region in which the constraint is not violated is nonempty, the maximum value of the objective must be achieved on one of the vertices of the multidimensional polyhedron formed by slicing off the portion of the unit simplex that violates the constraint. These vertices represent two types of random variables: (a) degenerate distributions with all of the mass on one value of $x$, and (b) two-point distributions that satisfy the constraint with equality.

This result is related to the well-established fact that condition $E f_{2}(\tilde{x}) \leq$ $E f_{2}\left(x_{0}\right)$ holds for any $\tilde{x}$ if and only if it holds for any degenerate (one-point) random variable. Introducing a condition on $\tilde{x}$, in particular that $\tilde{x}$ must satisfy $E f_{1}(\tilde{x}) \leq E f_{1}\left(x_{0}\right)$, makes it necessary to consider not only degenerate random variables, but also all two-point random variables.

The Diffidence Theorem is a consequence of Lemma 1. To escape triviality, assume that there exists at least one $x \in R$ such that $f_{1}(x) \leq E f_{1}\left(x_{0}\right)$.

\footnotetext{
${ }^{1}$ It is easy to check that $x_{0}$ can be replaced with a reference random variable $\tilde{\omega}$ without affecting the proof of the Diffidence Theorem, but the key applications we have in mind in this paper have a nonstochastic initial wealth. Replacing $x_{0}$ with $\tilde{\omega}$ would, however, affect the proof of Corollary 1 below.

${ }^{2}$ The proof of Theorem 3 in Pratt and Zeckhauser (1987) is based on the same basic idea.
} 
Theorem 1 (Diffidence Theorem) Condition (1) for any random variable $\tilde{x}$ with support in $[a, b]$ is equivalent to the condition that there exists an $m \in R_{+} \cup\{+\infty\}$ such that

$$
\forall x \in[a, b]: \quad f_{2}(x)-E f_{2}\left(x_{0}\right) \leq m\left[f_{1}(x)-E f_{1}\left(x_{0}\right)\right] .
$$

Proof: Notice first that the sufficiency of (4) is obvious. Turning to necessity, if $f_{1}$ reaches a global maximum at $x_{0}$, then the "if" clause in Condition (1) is true for any random variable, $E f_{2}(\tilde{x}) \leq E f_{2}\left(x_{0}\right)$ must hold for any $\tilde{x}$ and Condition (4) holds with $m=0$. If $f_{1}$ reaches a global minimum at $x_{0}$, then Condition (4) holds with $m=+\infty$. (Wherever $f_{1}(x)-f_{1}\left(x_{0}\right)=0$, Condition (1) for degenerate risks implies $f_{2}(x)-f\left(x_{0}\right) \leq 0$. Elsewhere, any possible value of $f_{2}(x)-f_{2}\left(x_{0}\right)$, satisfies $f_{2}(x)-f_{2}\left(x_{0}\right) \leq+\infty\left(f_{1}(x)-f_{1}\left(x_{0}\right)\right)$.) For necessity when $f_{1}$ is neither a global minimum nor a global maximum at $w$, let $\phi_{1}$ and $\phi_{2}$ denote $E f_{1}\left(x_{0}\right)$ and $E f_{2}\left(x_{0}\right)$, respectively. By the compactness of the simplex and the nontriviality assumption, a solution exists to program (3). So, considering two-point risks, we know that Condition (1) implies that $\forall x_{1}, x_{2} \in[a, b], \forall p \in[0,1]$ :

$$
p f_{1}\left(x_{1}\right)+(1-p) f_{1}\left(x_{2}\right)=\phi_{1} \quad \Longrightarrow \quad p f_{2}\left(x_{1}\right)+(1-p) f_{2}\left(x_{2}\right) \leq \phi_{2} .
$$

Consider an $x_{1}$ for which $f\left(x_{1}\right) \neq f\left(x_{0}\right)$. Suppose without loss of generality that $f_{1}\left(x_{1}\right)<\phi_{1}$ and $f_{1}\left(x_{2}\right)>\phi_{1}$. The first condition in (5) is equivalent to

$$
p=\frac{\phi_{1}-f_{1}\left(x_{2}\right)}{f_{1}\left(x_{1}\right)-f_{1}\left(x_{2}\right)} .
$$

Substituting $p$ in the second condition of (5) makes it equivalent to

$$
\left(\phi_{1}-f_{1}\left(x_{2}\right)\right)\left(f_{2}\left(x_{1}\right)-\phi_{2}\right) \geq\left(\phi_{1}-f_{1}\left(x_{1}\right)\right)\left(f_{2}\left(x_{2}\right)-\phi_{2}\right),
$$

for any $x_{1}$ and $x_{2}$ in $[a, b]$ such that $f_{1}\left(x_{1}\right)<\phi_{1}$ and $f_{1}\left(x_{2}\right)>\phi_{1}$. The above inequality is itself equivalent to

$$
\frac{f_{2}\left(x_{2}\right)-\phi_{2}}{f_{1}\left(x_{2}\right)-\phi_{1}} \leq m \leq \frac{f_{2}\left(x_{1}\right)-\phi_{2}}{f_{1}\left(x_{1}\right)-\phi_{1}},
$$

for some real number $m$ and for any $x_{1}$ and $x_{2}$ in $[a, b]$ such that $f_{1}\left(x_{1}\right)<\phi_{1}$ and $f_{1}\left(x_{2}\right)>\phi_{1}$. This is in turn equivalent to Condition (4) without the sign condition on $m$ (and we dealt above with the only case in which $m=+\infty$ is needed). The condition $m \geq 0$ is required to guarantee that Condition (4) is satisfied for all one-point random variables. 
It is noteworthy that Condition (4), with $m$ positive or negative, forces Condition (1) to hold for all two-point random variables $\tilde{x}$ that satisfy condition $E f_{1}(\tilde{x}) \leq E f_{1}\left(x_{0}\right)$ as an equality. The other condition $-m$ nonnegative - forces condition (1) for all degenerate (one-point) random variables. It is thus easy to verify that if the first inequality in (1) is replaced by an equality, the conclusion for necessity is the same except that $m$ can be any real number. ${ }^{3}$ The arguments above still apply, except in the case when $f_{1}$ reaches a maximum at $x_{0}$. In that case, $m=-\infty$ works. Thus, if Condition (1) is replaced by

$$
\forall \tilde{x}: \quad E f_{1}(\tilde{x})=E f_{1}\left(x_{0}\right) \Longrightarrow E f_{2}(\tilde{x}) \leq E f_{2}\left(x_{0}\right),
$$

the Diffidence Theorem for the case of an equality antecedent states that there exists an $m \in R \cup\{+\infty\} \cup\{-\infty\}$

$$
\forall x \in[a, b]: \quad f_{2}(x)-E f_{2}\left(x_{0}\right) \leq m\left[f_{1}(x)-E f_{1}\left(x_{0}\right)\right] .
$$

The marginal product of the Diffidence Theorem relative to the Lemma is that given the Diffidence Theorem, one only has to verify a unidimensional condition. But the cost is the necessity of looking for an $m$ that satisfies it. An important additional simplification can be obtained if $f_{1}$ and $f_{2}$ are differentiable at $x_{0}$, and $f_{1}^{\prime}\left(x_{0}\right) \neq 0$. In that case there is a unique candidate for $m$ identified in the following Corollary.

Corollary 1 If $f_{1}^{\prime}\left(x_{0}\right) \neq 0$ and $f_{2}^{\prime}\left(x_{0}\right)$ exists, the unique candidate for $m$ in (4) is

$$
m=\frac{f_{2}^{\prime}\left(x_{0}\right)}{f_{1}^{\prime}\left(x_{0}\right)} .
$$

If $f_{1}$ and $f_{2}$ are twice differentiable at $w$, a necessary condition for (1) is

$$
f_{2}^{\prime \prime}\left(x_{0}\right) \leq \frac{f_{2}^{\prime}\left(x_{0}\right)}{f_{1}^{\prime}\left(x_{0}\right)} f_{1}^{\prime \prime}\left(x_{0}\right) .
$$

Proof: Condition (4) means that function $\xi(x)$ must be nonpositive, with

$$
\xi(x)=f_{2}(x)-f_{2}\left(x_{0}\right)-m\left[f_{1}(x)-f_{1}\left(x_{0}\right)\right] .
$$

But $\xi\left(x_{0}\right)=0$, so the global maximum of $\xi$ must be at $w$. The necessary condition for a maximum must hold: $f_{2}^{\prime}\left(x_{0}\right)-m f_{1}^{\prime}\left(x_{0}\right)=0$, yielding the first

\footnotetext{
${ }^{3}$ Also, the change of the direction of the first and/or the second inequality in (1) can easily be considered by replacing $f_{i}$ with $-f_{i}$
} 


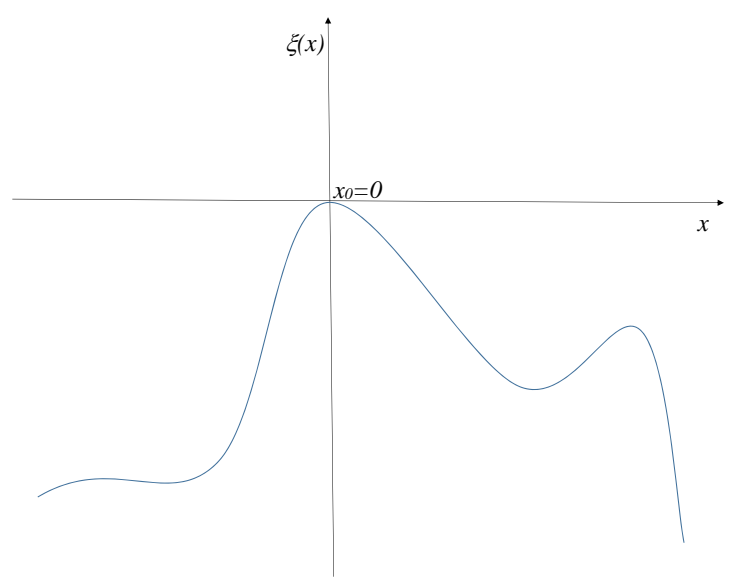

Figure 1: Local Diffidence Conditions: If $\xi(x)$ must be non-positive everywhere, and if $\xi\left(x_{0}\right)$ is zero, then $\xi^{\prime}\left(x_{0}\right)$ must be zero and $\xi^{\prime \prime}\left(x_{0}\right)$ must be non-positive. These local necessary conditions are generally not sufficient for $\xi$ to be non-positive everywhere.

result. Condition (10) is a direct consequence of the second-order condition $\xi^{\prime \prime}\left(x_{0}\right) \leq 0$. This concludes the proof, whose basic idea is described in Figure 1.

Remark: If $f_{1}^{\prime}\left(x_{0}\right)=0$, then $f_{2}^{\prime}\left(x_{0}\right)-m f_{1}^{\prime}\left(x_{0}\right)=0$ implies that $f_{2}^{\prime}\left(x_{0}\right)=0$ as well. But then any $m$ satisfies $f_{2}^{\prime}\left(x_{0}\right)-m f_{1}^{\prime}\left(x_{0}\right)=0$, so the Corollary provides no guidance for the value of $m$ when $f_{1}^{\prime}\left(x_{0}\right)=0$.

Because $m$ has to be nonnegative, Condition (9) indicates that $f_{1}^{\prime}\left(x_{0}\right)$ and $f_{2}^{\prime}\left(x_{0}\right)$ must have the same sign. In fact, this is a "local" necessary condition guaranteeing that property (1) holds for all degenerate random variables close to $w$. Notice also that necessary condition (10) is necessary and sufficient for (1) to hold for any small risk whose support is in the neighborhood of $w$.

In general, the problem faced by researchers is a bit more complicated than (1). More specifically, functions $f_{i}$ may depend upon a parameter (or parameter vector) $w$, leading to the problem

$$
\forall \tilde{x}: \quad E F_{1}(w, \tilde{x}) \leq F_{1}\left(w, x_{0}\right) \Longrightarrow E F_{2}(w, \tilde{x}) \leq F_{2}\left(w, x_{0}\right) .
$$


For most of the applications below, $w$ is a scalar that can be interpreted as initial wealth. When allowing for a parameter or parameter vector $w$, it is usually convenient to focus on functions for which $x_{0}=0: f_{i}(w, x)=$ $F_{i}\left(w, x-x_{0}\right)$. Then the condition above becomes

$$
\forall \tilde{x}: \quad E f_{1}(w, \tilde{x}) \leq f_{1}(w, 0) \Longrightarrow E f_{2}(w, \tilde{x}) \leq f_{2}(w, 0) .
$$

A direct extension of the Diffidence Theorem is that condition (12) has the following necessary and sufficient condition

$$
\forall x \in[a, b]: \quad f_{2}(w, x)-f_{2}(w, 0) \leq \frac{\frac{\partial f_{2}}{\partial x}(w, 0)}{\frac{\partial f_{1}}{\partial x}(w, 0)}\left[f_{1}(w, x)-f_{1}(w, 0)\right] .
$$

We refer to this condition as the "central" necessary and sufficient condition associated with parameter $w$. Closely connected to this condition are two "local" necessary conditions:

$$
\frac{\frac{\partial f_{2}}{\partial x}(w, 0)}{\frac{\partial f_{1}}{\partial x}(w, 0)} \geq 0
$$

and

$$
\frac{\partial^{2} f_{2}}{\partial x^{2}}(w, 0) \leq \frac{\frac{\partial f_{2}}{\partial x}(w, 0)}{\frac{\partial f_{1}}{\partial x}(w, 0)} \frac{\partial^{2} f_{1}}{\partial x^{2}}(w, 0)
$$

When property (12) is required to hold for all values of the parameter $w$, Condition (13) becomes the global necessary and sufficient condition. This global necessary and sufficient condition (13) may be complex to verify. It requires that a bivariate function be uniformly nonpositive.

In order to study the global necessary and sufficient condition (13) and necessary conditions (14) and (15), from here on in the paper, we will assume that $f_{1}$ and $f_{2}$ are twice differentiable, unless stated otherwise. If $f_{1}$ or $f_{2}$ might not be differentiable, one must return to the Diffidence Theorem itself, with less guidance about the values of $m$ that will work for each 0 . If $f_{1}$ and $f_{2}$ can be assumed to be differentiable, but may not be twice differentiable, Condition (15) must be replaced by a statement that

$$
f_{2}(x)-\frac{\frac{\partial f_{2}}{\partial x}(w, 0)}{\frac{\partial f_{1}}{\partial x}(w, 0)} f_{1}(x)
$$

is locally concave in the neighborhood of $w$. 


\section{Applications of the Diffidence Theorem}

There are examples of problems of type (12) for which the combination of the univariate necessary conditions (14) and (15) is sufficient. This leads to a characterization of the solution that is simpler than the bivariate characterization (13). These examples are gathered in the first part of this section.

\subsection{Applications with a univariate necessary and sufficient condition}

The celebrated results of Pratt (1964) are directly relevant to applications of the Diffidence Theorem in which the functions $f_{i}(w, x)$ take the additive form $g_{i}(w+x)$, i.e., when $w$ and $x$ are perfect substitutes. For these cases, we obtain the following result, a restatement of the main result of Pratt (1964).

Proposition 1 The following two conditions are equivalent:

1. For all $w$ and for all $\tilde{x}$ such that the support of $w+\tilde{x}$ is in the joint domain of $\left(g_{1}, g_{2}\right)$,

$$
E g_{1}(w+\tilde{x}) \leq g_{1}\left(x_{0}\right) \Longrightarrow E g_{2}(w+\tilde{x}) \leq g_{2}(w) .
$$

2. Function $\varphi$ is increasing and concave, where $\varphi$ is such that $\varphi\left(g_{1}(t)\right)=$ $g_{2}(t)$ for all $t$ in the joint domain of $\left(g_{1}, g_{2}\right)$.

The property that $\varphi$ is an increasing function is equivalent to the necessary condition (14) in this additive case. Similarly, given the twicedifferentiability we have assumed, the condition that $\varphi$ is concave is equivalent to Condition (15), which can be rewritten as:

$$
\forall w: \quad g_{2}^{\prime \prime}(w) \leq \frac{g_{2}^{\prime}(w)}{g_{1}^{\prime}(w)} g_{1}^{\prime \prime}(w)
$$

This shows that in this additive case, the Diffidence Theorem, when applied at all wealth levels, simplifies to the classical condition that $g_{2}$ be more concave than $g_{1}$ in the sense of Arrow-Pratt. As explained by Pratt [1964], the necessary and sufficient condition "in the small" obtained from comparing the indices of absolute concavity $-g_{i}^{\prime \prime} / g_{i}^{\prime}$ is also necessary and sufficient "in the large".

It is easy to show that when the first inequality in condition (17) is replaced by an equality, the necessary and sufficient condition simplifies to 
$\varphi$ being concave. The monotonicity of $\varphi$ is not required in that case. In the next subsections, we show what the Diffidence Theorem adds to Arrow-Pratt theory.

\subsubsection{Central diffidence, central risk aversion and global risk aver- sion}

Probably the simplest application of the Diffidence Theorem is the characterization of "diffidence" itself. An agent is centrally diffident around $w$ if and only if:

$$
\forall \tilde{x} \quad E \tilde{x}=0 \quad \Longrightarrow \quad E u(w+\tilde{x}) \leq u(w),
$$

for all $\tilde{x}$ and a particular $w$. That is, an agent is centrally diffident around $w$ if the agent would reject any mean zero risk added to that initial wealth level $w$. An agent is globally diffident if this holds for all $w$. Assuming differentiability of $u$, the necessary and sufficient condition for central diffidence around $w$ is that for some real number $m$,

$$
u(w+x)-u(w) \leq m x
$$

(Note that the function $x$ has no global maximum or minimum.) Because $E \tilde{x}=0$ is an equation rather than an inequality, there is no restriction on the sign of $m$. If $u$ is differentiable, $m=u^{\prime}(w)$ and the condition for central diffidence around $w$ becomes

$$
\forall x \quad u(w+x)-u(w) \leq u^{\prime}(w) x
$$

The power of the method here is that the universal quantifier $\forall x$ in (21) is a quantifier only over all real numbers $x$, not over all distributions $\tilde{x}$. Geometrically, this condition says that the utility function lies below its tangent line at $w$. This has the local necessary condition $u^{\prime \prime}(x) \leq 0$, as illustrated in Figure 2. Thus, if central diffidence holds for all possible initial wealth levels $w$, then the utility function must be concave and therefore exhibits global risk aversion in the usual sense.

We define central risk aversion around $w$ by the stronger property that an agent always wants less in absolute value of the amount $\alpha$ of a mean-zero risk in a portfolio problem. Since $\frac{\partial}{\partial \alpha} E u(w+\alpha \tilde{x})=E \tilde{x} u^{\prime}(w+\tilde{x})$, saying that this derivative with respect to $\alpha$ has the opposite sign from $\alpha$ itself when $\tilde{x}=0$ can be written

$$
\forall \tilde{x} \quad E \tilde{x}=0 \quad \Longrightarrow \quad \alpha E \tilde{x} u^{\prime}(w+\alpha \tilde{x}) \leq 0 .
$$




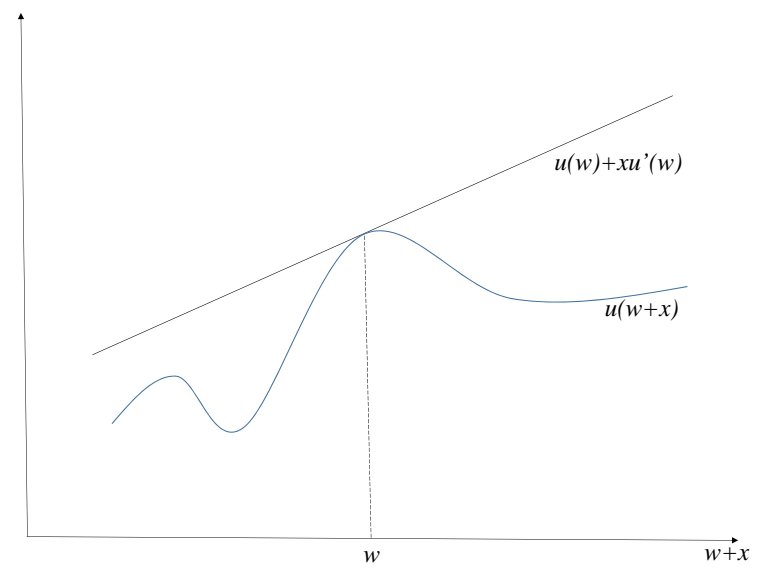

Figure 2: An example of a utility function $u$ that is central diffident around wealth level $w$, but is not concave. Concavity is necessary only locally around $w$.

The powerful universal quantifier over all distributions $\tilde{x}$ allows one to normalize $\alpha$ to unity. Thus, central risk aversion around $w$ has the necessary and sufficient condition

$$
\forall x \quad x u^{\prime}(w+x) \leq x u^{\prime}(w)
$$

Marginal utility is lower than to the right of $w$ than at $w$ and higher to the left of $w$ than at $w$, as is illustrated in Figure 3. This also has the local necessary condition $u^{\prime \prime}(x) \leq 0$. Thus, if central risk aversion holds for all possible initial wealth levels $w$ it also implies concavity and therefore global risk aversion in the usual sense.

How are central diffidence and central risk aversion related to one another when they hold only for a particular initial wealth $w$ ? First, if the initial wealth level is known, these are very useful properties for applications. Global risk aversion is often stronger than necessary. To guarantee that an agent will reject all mean-zero risks given a particular initial wealth $w$, it is necessary and sufficient for the utility curve $u$ to lie entirely below its tangent line at $w$. To guarantee that an agent will always want less (in absolute value) of a mean-zero risk, it is necessary and sufficient for marginal utility to be above $u^{\prime}(w)$ to the left of $w$ and below $u^{\prime}(w)$ to the right of $w$. Although either of these conditions requires that $u^{\prime \prime}(w)$ be 


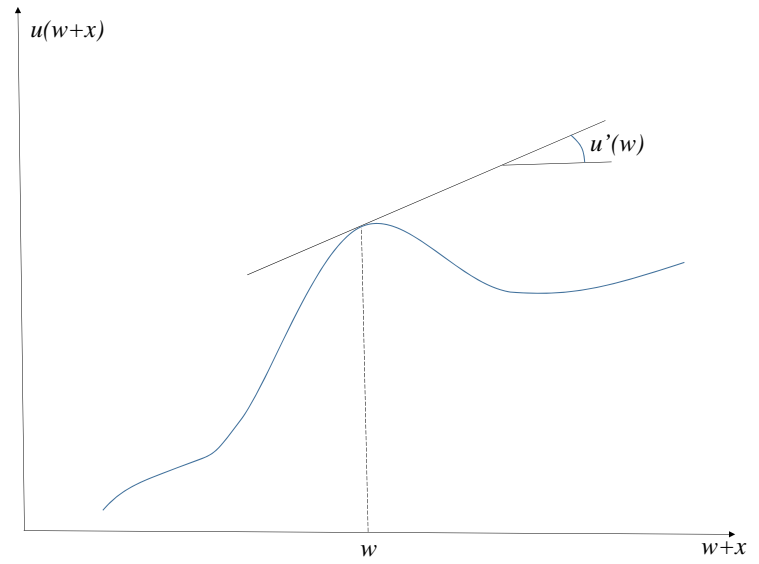

Figure 3: An example of a utility function $u$ that is centrally risk-averse around wealth level $w$, but is not concave. Concavity is necessary only locally around $w$.

negative, and therefore $u^{\prime}(w)$ decreasing, in the neighborhood of $w$, they are less demanding than global risk aversion.

Second, fixing the initial wealth $w$, central risk aversion implies central diffidence but not vice versa. That is, central risk aversion is a stronger property than central diffidence. This can be shown by integrating the Necessary and Sufficient Condition (NSC) for central risk aversion:

Central Risk Aversion around $w \Longrightarrow$ Central Diffidence around $w$ :

$$
u(w+x)-u(w)-u^{\prime}(w) x=\int_{0}^{x}\left[u^{\prime}(\xi)-u^{\prime}(w)\right] d \xi \leq 0 .
$$

Geometrically, central diffidence around $w$ requires that the utility function lie below its tangent line at $w$. Central risk aversion around $w$ requires that the the gap between the utility function and the tangent at $w$ is always increasing as one moves away from $w$. Clearly, the requirement that the gap $u(w)+u^{\prime}(w) x-u(w+x)$ is increasing as one moves away from $w$ is a stronger condition than the requirement that the gap $u(w)+u^{\prime}(w) x-u(w+x)$ be positive. Hence, central risk aversion is a stronger property than central diffidence. Central risk aversion implies central diffidence, but central diffidence does not imply central risk aversion. 


\subsubsection{Greater diffidence and greater risk aversion}

For some applications globally greater risk aversion is more than is needed: it is sometimes enough to know that an agent will act more risk averse from a given starting wealth. This comes up, for example, in studying proper risk aversion (Pratt and Zeckhauser, 1987) and standard risk aversion (Kimball, 1993). Proper risk aversion is when an undesirable independent background risk always makes an agent reject more risks. Standard risk aversion is when an independent background risk that raises expected marginal utility always makes an agent reject more risks. The interesting thing about these properties is that an independent background risk being undesirable or raising expected marginal utility can be true at some initial wealth levels and not others. So, both proper risk aversion and standard risk aversion are saying that a particular set of independent background risks will make the derived utility function obtained by taking an expectation over the background risk centrally more diffident around some values of starting wealth than the original utility function with no background risk. This aspect of (Pratt and Zeckhauser, 1987) and (Kimball, 1993) piqued our interest in central properties and played a key role in the historical origins of this paper.

By contrast, risk vulnerability (Gollier and Pratt, 1996) says that a mean-zero independent background risk will make an agent reject more risks. A mean-zero background risk is mean-zero regardless of the starting wealth, so risk vulnerability implies that a mean-zero background risk will make the derived utility function obtained by taking an expectation over the background risk globally more diffident in the usual Arrow-Pratt sense.

The logical relationships between central properties are different than between global properties. In particular, globally greater diffidence and globally greater risk aversion are the same thing and equivalent to the familiar Arrow-Pratt notion of globally greater risk aversion. By contrast, for a given initial wealth centrally greater risk aversion implies centrally greater diffidence but not vice versa. That is, centrally greater risk aversion is a stronger property than centrally greater diffidence.

In accordance with the definitions of central diffidence and central risk aversion above, define centrally greater diffidence and centrally greater risk aversion as follows. Paralleling the definition of central diffidence itself, $u_{2}$ centrally more diffident than $u_{1}$ around the initial wealth $w$ is defined by:

$$
\forall \tilde{x}: \quad E u_{1}(w+\tilde{x}) \leq u_{1}(w) \quad \Longrightarrow \quad E u_{2}(w+\tilde{x}) \leq u_{2}(w) .
$$

That is, agent 2 rejects $w+\tilde{x}$ in favor of $w$ for more risks $\tilde{x}$ than agent 1 
does. Paralleling the definition of central risk aversion itself, $u_{2}$ centrally more risk averse than $u_{1}$ around the initial wealth $w$ is defined by:

$$
\forall \tilde{x}: \quad E \tilde{x} u_{1}^{\prime}(w+\tilde{x}) \leq 0 \Longrightarrow E \tilde{x} u_{2}^{\prime}(w+\tilde{x}) \leq 0 .
$$

Assuming concavity of both $u_{1}$ and $u_{2}$, the economic interpretation of centrally greater risk aversion is that agent 2 would always choose an amount $\alpha$ of the risk $\tilde{x}$ that is smaller in absolute value than agent $1 .{ }^{4}$

Using the Diffidence Theorem, one can obtain the following necessary and sufficient conditions for centrally greater diffidence and centrally greater risk aversion. For centrally greater diffidence (assuming both $u_{1}^{\prime}$ and $u_{2}^{\prime}$ are strictly positive) the central necessary and sufficient condition is:

$$
\forall x: \frac{u_{2}(w+x)-u_{2}(w)}{u_{2}^{\prime}(w)} \leq \frac{u_{1}(w+x)-u_{1}(w)}{u_{1}^{\prime}(w)}
$$

If one normalizes $u_{1}$ and $u_{2}$ so that $u_{1}(w)=u_{2}(w)$ and $u_{1}^{\prime}(w)=u_{2}^{\prime}(w)$, centrally greater risk aversion means that the $u_{2}$-curve is tangent to $u_{1}$ at $w$, but everywhere weakly below the $u_{1}$-curve, as illustrated in Figure 4 . For centrally greater risk aversion, the central NSC is:

$$
\forall x: x \frac{u_{2}^{\prime}(w+x)}{u_{2}^{\prime}(w)} \leq x \frac{u_{1}^{\prime}(w+x)}{u_{1}^{\prime}(w)}
$$

With the normalization $u_{1}^{\prime}(w)=u_{2}^{\prime}(w)$ (one component of the normalization above), this is single crossing of marginal utility.

\footnotetext{
${ }^{4}$ Without the assumption of concavity, the techniques of monotone comparative statics would still lead to a set of optimizing values of $\alpha$ for $u_{2}$ that are smaller in absolute value than the set of optimizing values of $\alpha$ for $u_{1}$.
} 


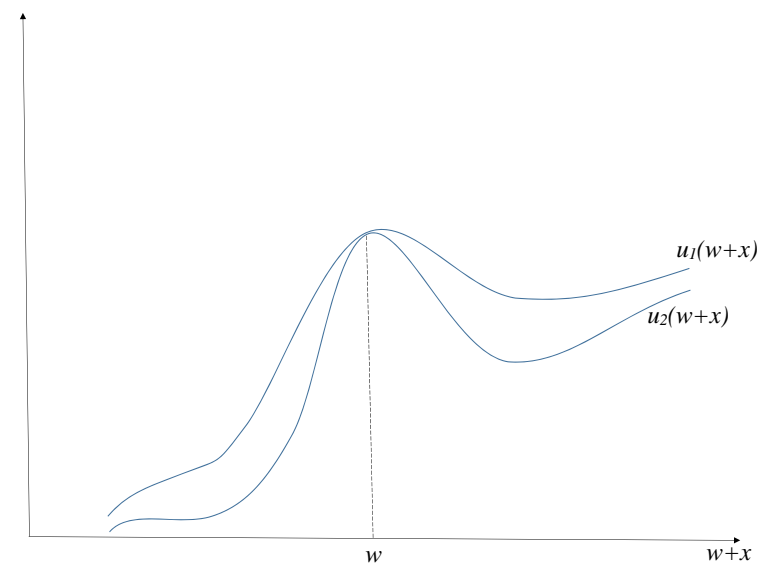

Figure 4: An example of a utility function $u_{2}$ that is centrally more diffident than $u_{1}$ around wealth level $w$, but is not globally more concave than it. Greater concavity is necessary only locally around $w$.

Given this characterization of the necessary and sufficient conditions for centrally greater diffidence and centrally greater risk aversion, integration of the NSC for centrally greater risk aversion yields the NSC for centrally greater diffidence, showing that centrally greater risk aversion implies centrally greater diffidence:

$$
\begin{gathered}
\frac{u_{2}(w+x)-u_{2}(w)}{u_{2}^{\prime}(w)}-\frac{u_{1}(w+x)-u_{1}(w)}{u_{1}^{\prime}(w)}=\int_{0}^{x}\left\{\left[\frac{u_{2}^{\prime}(w+\xi)}{u_{2}^{\prime}(w)}\right]-\left[\frac{u_{1}^{\prime}(w+\xi)}{u_{1}^{\prime}(w)}\right]\right\} d \xi \\
\leq 0
\end{gathered}
$$

Here the sign consequences of the order of integration cancel out the sign of $\left[u_{2}^{\prime}(w+\xi) / u_{2}^{\prime}(w)\right]-\left[u_{1}^{\prime}(w+\xi) / u_{1}^{\prime}(w)\right]$. The converse is false: $\left[\left(u_{2}(w+x)-\right.\right.$ $\left.\left.u_{2}(w)\right) / u_{2}^{\prime}(w)\right]-\left[\left(u_{1}(w+x)-u_{1}(w)\right) / u_{1}^{\prime}(w)\right]$ can be everywhere negative, as required for greater central diffidence, without being everywhere increasing to the left (when $x \leq 0$ ) and everywhere decreasing to the right (when $x \geq 0$ ) as required for centrally greater risk aversion. Of course, being equal to zero at $x=0$ and everywhere below requires being increasing to the left of zero in a neighborhood of $x=0$ and increasing to the right in a neighborhood of $x=0$, so the local necessary conditions (NCs) for centrally greater diffidence and centrally greater risk aversion are identical: 


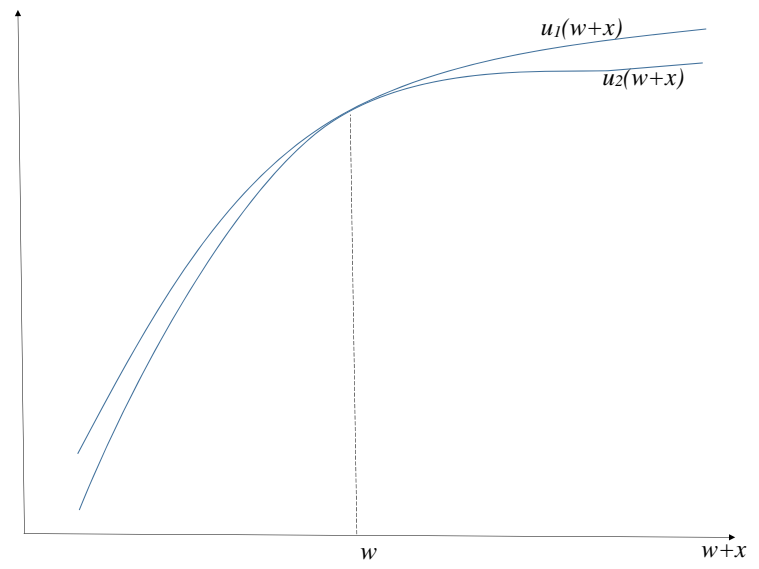

Figure 5: An example of a utility function $u_{2}$ that is centrally more riskaverse than $u_{1}$ around wealth level $w$, but is not globally more concave than $u_{1}$. Greater concavity is necessary only locally around $w$.

$$
\frac{u_{2}^{\prime \prime}(w)}{u_{2}^{\prime}(w)} \leq \frac{u_{1}^{\prime \prime}(w)}{u_{1}^{\prime}(w)}
$$

or

$$
A_{2}(w) \geq A_{1}(w)
$$

where $A_{i}(w)=-u_{i}^{\prime \prime}(w) / u_{i}^{\prime}(w)$ is the index of absolute risk aversion of individual $i$. Thus, even though greater central risk aversion is a stronger property, when restricted to a neighborhood of $x=0$, it leads to the same local property. From here on, we will refer to that local property as locally greater risk aversion.

Locally greater risk aversion in the neighborhood of a particular initial wealth $w$ clearly does not imply centrally greater diffidence-let alone greater central risk aversion-around that initial wealth. But if one requires locally greater risk aversion to hold for all initial wealth levels $w$, it implies centrally greater risk aversion for all initial wealth levels. Since

$\left[\ln \left(u_{2}^{\prime}(w+x)\right)-\ln \left(u_{2}^{\prime}(w)\right)\right]-\left[\ln \left(u_{1}^{\prime}(w+x)\right)-\ln \left(u_{1}^{\prime}(w)\right)\right]=\int_{0}^{x}\left\{\left[\frac{u_{2}^{\prime \prime}(\xi)}{u_{2}^{\prime}(\xi)}\right]-\left[\frac{u_{1}^{\prime \prime}(\xi)}{u_{1}^{\prime \prime}(\xi)}\right]\right\} d \xi$, 
the global Arrow-Pratt condition $A_{2}(w) \geq A_{1}(w)$, or equivalently, $u_{2}^{\prime \prime}(w) / u_{2}^{\prime}(w) \leq$ $u_{1}^{\prime \prime}(w) / u_{2}^{\prime}(w)$ implies

$$
\begin{array}{ll}
\forall x \geq 0: & {\left[\ln \left(u_{2}^{\prime}(w+x)\right)-\ln \left(u_{2}^{\prime}(w)\right)\right]-\left[\ln \left(u_{1}^{\prime}(w+x)\right)-\ln \left(u_{1}^{\prime}(w)\right)\right] \leq 0} \\
\forall x \leq 0: & {\left[\ln \left(u_{2}^{\prime}(w+x)\right)-\ln \left(u_{2}^{\prime}(w)\right)\right]-\left[\ln \left(u_{1}^{\prime}(w+x)\right)-\ln \left(u_{1}^{\prime}(w)\right)\right] \geq 0 .}
\end{array}
$$

This is equivalent to the condition for greater central risk aversion:

$$
\begin{array}{ll}
\forall x \geq 0: & \frac{\left.u_{2}^{\prime}(w+x)\right)}{u_{2}^{\prime}(w)} \leq \frac{\left.u_{1}^{\prime}(w+x)\right)}{u_{1}^{\prime}(w)} \\
\forall x \leq 0: & \frac{\left.u_{2}^{\prime}(w+x)\right)}{u_{2}^{\prime}(w)} \geq \frac{\left.u_{1}^{\prime}(w+x)\right)}{u_{1}^{\prime}(w)} .
\end{array}
$$

Let us summarize the results about locally greater risk aversion, centrally greater diffidence, centrally greater risk aversion and globally greater risk aversion. Think of locally greater risk aversion, centrally greater risk aversion and centrally greater diffidence arranged in a triangle. For a particular initial wealth $w$, centrally greater risk aversion implies centrally greater diffidence, which in turn implies locally greater risk aversion. But locally greater risk aversion does not imply either centrally greater risk aversion or centrally greater diffidence. And centrally greater diffidence does not imply centrally greater risk aversion.

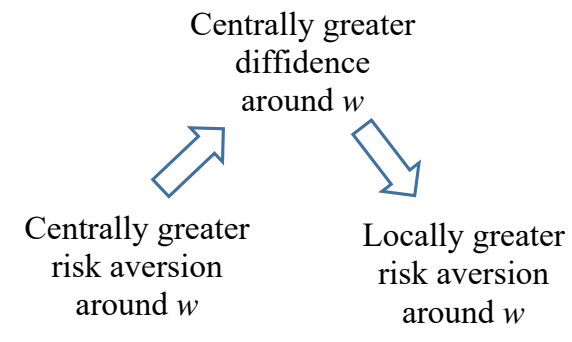

Now, add the universal quantifier "for all initial wealth levels $w$." Locally greater risk aversion in the neighborhood of all initial wealth levels $w$ implies centrally greater risk aversion around every initial wealth level $w$, which implies centrally greater diffidence around every initial wealth level $w$, which in turn implies greater local risk aversion in the neighborhood of every initial wealth level $w$. Thus, all three are equivalent and can be given the same name: "globally greater risk aversion." 


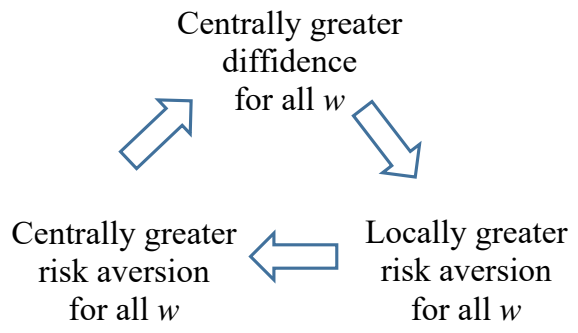

The implications on two sides of the triangle work initial wealth level by initial wealth level; but the implication from locally greater risk aversion in the neighborhood of every initial wealth to centrally great risk aversion around every initial wealth requires, in effect, stitching together locally greater risk aversion at every initial wealth to get centrally greater risk aversion around even one initial wealth level. Fortunately, the stitching together of locally greater risk aversion conditions to get centrally greater risk aversion around one initial wealth level works similarly to get centrally greater risk aversion around any initial wealth level.

\subsubsection{Prudence and central prudence}

Eeckhoudt and Schlesinger (2006) define an atemporal concept of prudence by considering two harms in the form of a sure loss $\ell$ and a zero-mean risk $\tilde{x}$. A prudent individual "prefers to receive one of the two harms for certain, with the only uncertainty being about which one is received, as opposed to a 50-50 chance of receiving both harms simultaneously or receiving neither" ${ }^{5}$ This is the case if the utility premium $z(w)=E u(w+\tilde{x})-u(w)$ is increasing. Thus, prudence can be defined as follows:

$$
E \tilde{x}=0 \quad \Longrightarrow \quad E u^{\prime}(w+\tilde{x}) \geq u^{\prime}(w),
$$

for all $\tilde{x}$ and all $w$. It is centrally prudent at $w$ if the above condition is true for all $\tilde{x}$ for this specific $w$. Notice that, following Kimball (1990), prudence can more traditionally be defined by requiring that consumers reduce their current consumption when a zero-mean risk is added to future income. Applying the same analysis as for risk aversion, we obtain the

\footnotetext{
${ }^{5}$ An earlier version of the same definition is provided by Eeckhoudt, Gollier and Schneider [1995].
} 
following two characterizations:

$$
\begin{aligned}
& \text { Prudence: } \quad u^{\prime} \text { convex } \\
& \text { Central Prudence: } \quad \forall x: u^{\prime}(w+x)-u^{\prime}(w) \geq u^{\prime \prime}(w) x \text {. }
\end{aligned}
$$

Although central prudence requires that $u^{\prime \prime \prime}(w)$ be positive, it is less demanding than prudence.

Central prudence looks like diffidence, one derivative up. By analogy to central risk aversion, one could also define "scale-up prudence" by an increase in the scale of a mean-zero risk raising expected marginal utility. (Central risk aversion could have been called "central scale-up diffidence.") The mathematical condition for central scale-up prudence is:

$$
\text { Central Scale-Up Prudence: } x u^{\prime \prime}(w+x) \geq x u^{\prime \prime}(w)
$$

That is, the second derivative of the utility function is algebraically higher to the right of $w$ and lower to the left of $w$ than at $w$. If $u^{\prime \prime}(w+x)<0$, that means $u^{\prime \prime}$ is closer to zero to the right of $w$ and further from zero to the left of $w$. When they hold globally, prudence and scale-up prudence are equivalent, but for a particular starting wealth $w$ they are distinct concepts.

\subsubsection{Greater prudence and centrally greater prudence}

Under what mathematical condition on the two utility functions does any risk that increases the precautionary saving (or utility premium) of agent $u_{1}$ also increases the precautionary saving (or utility premium) of agent $u_{2}$ ? In the discounted expected utility model in which individual $i$ selects the consumption plan $\left(w_{0}, w\right)$ to maximize $v\left(w_{0}\right)+u_{i}(w)$ under some intertemporal budget constraint, this is true if and only if

$$
\forall w, \tilde{x}: \quad E u_{1}^{\prime}(w+\tilde{x}) \geq u_{1}^{\prime}(w) \quad \Longrightarrow \quad E u_{2}^{\prime}(w+\tilde{x}) \geq u_{2}^{\prime}(w)
$$

where $w$ can be interpreted as optimal future consumption in the absence of risk. Depending upon whether $w$ is arbitrary or fixed, this condition defines the concept of greater prudence or centrally greater prudence. Define the index of absolute prudence of agent $i$ by $P_{i}(w)=-u_{i}^{\prime \prime \prime}(w) / u_{i}^{\prime \prime}(w)$. Assuming $u_{1}^{\prime \prime}(w)<0$ and $u_{2}^{\prime \prime}(w)<0$, these concepts can be characterized as follows:

$$
\begin{array}{rlrl}
\text { Greater Prudence: } & \forall w: P_{2}(w) & \geq P_{1}(w) \\
\text { Centrally Greater Prudence: } & \forall x: \frac{u_{2}^{\prime}(w+x)-u_{2}^{\prime}(w)}{u_{2}^{\prime \prime}(w)} \leq \frac{u_{1}^{\prime}(w+x)-u_{1}^{\prime}(w)}{u_{1}^{\prime \prime}(w)} .
\end{array}
$$


By analogy to centrally greater risk aversion, centrally greater scale-up prudence can be defined thus: if a small increase in the scale of a risk raises expected marginal utility for $u_{1}$, then that increase in scale also raises expected marginal utility for $u_{2}$. The relationship between centrally greater prudence and centrally greater scale-up prudence is analogous to the relationship between centrally greater diffidence and centrally greater risk aversion. ssuming $u_{1}^{\prime \prime}(w)<0$ and $u_{2}^{\prime \prime}(w)<0$, the mathematical condition for greater scale-up prudence is

$$
\text { Centrally Greater Scale-Up Prudence: } \forall x: x \frac{u_{2}^{\prime \prime}(w+x)}{u_{2}^{\prime \prime}(w)} \leq x \frac{u_{1}^{\prime \prime}(w+x)}{u_{1}^{\prime \prime}(w)} \text {. }
$$

\subsubsection{Decreasing absolute risk aversion}

There are several ways to define the concept of decreasing absolute risk aversion. One is that after a decrease in initial wealth an initially undesirable risk always remains undesirable. Another way to define decreasing absolute risk aversion is the condition that prudence is stronger than risk aversion, i.e. that any undesirable risk on future income reduces current consumption:

$$
\forall w, \tilde{x}: \quad E u_{1}(w+\tilde{x}) \leq u_{1}(w) \quad \Longrightarrow \quad E u_{1}^{\prime}(w+\tilde{x}) \geq u_{1}^{\prime}(w) .
$$

This problem is equivalent to problem (25) by defining $u_{2}=-u_{1}^{\prime}$. The same conclusion applies, with a necessary and sufficient condition that the index of absolute risk aversion for $-u_{1}^{\prime}$ be larger than the index of absolute risk aversion for $u_{1}$, i.e. $P_{1}(w) \geq A_{1}(w)$ for all $w$. It is easily seen that this is equivalent to decreasing absolute risk aversion of $u_{1}$ since $A_{1}^{\prime}(w)=$ $A_{1}(w)\left[A_{1}(w)-P_{1}(w)\right] \leq 0$.

Central decreasing absolute risk aversion can be defined by requiring that, given a particular starting point $w$ next period, any undesirable risk reduces current consumption-or alternatively, that beginning from starting wealth $w$, a small decrease in wealth leaves any initially undesirable risk still undesirable. The mathematical condition for central decreasing absolute risk aversion is that $-u_{1}^{\prime}$ is centrally more diffident around $w$ than $u_{1}$.

Central scale-up decreasing absolute risk aversion can be defined by requiring that, given a particular starting point $w$ next period, any undesirable increase in the scale of a risk also reduces current consumption-or alternatively, that beginning from starting wealth $w$, any initially undesirable scaling-up of a risk remains undesirable after a small decrease in initial 
wealth. The mathematical condition for central scale-up decreasing absolute risk aversion is that $-u_{1}^{\prime}$ must be centrally more risk averse around $w$ than $u_{1}$.

Dionne and Ingabire (2001) extend these results to the case of statedependent expected utility. In particular, they establish formal necessary and sufficient conditions in order to obtain prudence and decreasing absolute risk aversion when the utility function is state-specific.

\subsubsection{Decreasing absolute prudence}

Suppose that adding risk $\tilde{x}$ to future income $w$ does not affect current saving. Following Kimball [1993], we say that the individual has decreasing absolute prudence if an increase in $w$ implies that adding risk $\tilde{x}$ to $w$ induces the individual to save less. Technically, this is true iff ${ }^{6}$

$$
\forall w, \tilde{x}: \quad E u_{1}^{\prime}(w+\tilde{x})=u_{1}^{\prime}(w) \quad \Longrightarrow \quad E u_{1}^{\prime \prime}(w+\tilde{x}) \leq u_{1}^{\prime \prime}(w) .
$$

From Proposition 1, this condition holds if and only if $u_{1}^{\prime \prime}$ is more concave than $-u_{1}^{\prime}$. It is easy to verify that this condition is equivalent to $P_{1}$ being nonincreasing. One can also define central decreasing absolute prudence and central scale-up decreasing absolute prudence by analogy to central decreasing absolute risk aversion and central scale-up decreasing absolute risk aversion. The respective mathematical conditions are $u_{1}^{\prime \prime}$ being centrally more diffident than $-u_{1}^{\prime}$ around some initial level $w$ for central decreasing absolute prudence and $u_{1}^{\prime \prime}$ being centrally more risk averse than $-u_{1}^{\prime}$ around some initial level $w$ for central scale-up decreasing absolute prudence.

\subsubsection{Temperance and central temperance}

Eeckhoudt and Schlesinger (2006) also define an atemporal concept of temperance by using the same preference for the apportionment of harms, when the sure-loss harm is replaced by an independent zero-mean risk. Temperance is then defined by the concavity of the utility premium function $z_{1}$ :

$$
E \tilde{x}=0 \quad \Longrightarrow \quad E u_{1}^{\prime \prime}(w+\tilde{x}) \leq u_{1}^{\prime \prime}(w)
$$

for all $\tilde{x}$ and all $w$. A utility function is centrally temperate around $w$ if the above condition is true for all $\tilde{x}$ for this specific $w$. We leave the

\footnotetext{
${ }^{6}$ Gollier [1996] provides a simple interpretation of this condition which is based on how a marketable risk should be shared when an agent in the pool bears another nonmarketable risk.
} 
characterization of greater temperance, centrally greater temperance and centrally greater scale-up temperance as an exercise for interested readers: it looks like the characterization of globally greater prudence, centrally greater prudence and centrally greater scale-up prudence, one more derivative up.

\subsubsection{Effect of a new investment opportunity on consumption}

Suppose that consumers have access to a new risky asset whose net payoff will be delivered next period. Under what mathematical condition on the utility function does this new investment opportunity increase consumption and reduce saving today? ${ }^{7}$ Let $\tilde{x}$ denote this net payoff for one unit of this asset, and suppose without loss of generality that the optimal investment strategy is to purchase one unit of the asset. Then, this new investment opportunity reduces saving if and only the following condition holds:

$$
\forall \tilde{x}: \quad E \tilde{x} u^{\prime}(w+\tilde{x})=0 \quad \Longrightarrow \quad E u^{\prime}(w+\tilde{x}) \leq u^{\prime}(w),
$$

Applying the Diffidence Theorem with $f_{1}(x)=x u^{\prime}(w+x)$ and $f_{2}(x)=$ $u^{\prime}(w+x)-u^{\prime}(w)$ yields the following central necessary and sufficient condition for any given initial wealth $w:^{8}$

$$
\forall x: u^{\prime}(w+x)-u^{\prime}(w) \leq \frac{u^{\prime \prime}(w)}{u^{\prime}(w)} x u^{\prime}(w+x) .
$$

The local necessary condition is equivalent to $P(w) \leq 2 A(w)$. Observe that this condition is equivalent to $g(w)=1 / u^{\prime}(w)$ being convex. It implies that $g(w+x) \geq g(w)+x g^{\prime}(w)$ for all $w$ and $x$. This is equivalent to the sufficient condition (43). This is therefore another example in which the local condition holding at all wealth levels implies the global condition.

Suppose one confronted this problem, but did not immediately see that the condition $P(w) \leq 2 A(w)$ implied convexity of $1 / u^{\prime}(w)$. How should one proceed? Many courses in differential equations do not cover differential inequalities, so let us illustrate how to approach a differential inequality such as the local necessary condition here, assumed to hold for every starting point $w$. The trick is to replace the differential inequality of the local necessary condition with the corresponding differential equation, solve that differential equation, then go back and see if the solution to the differential

\footnotetext{
${ }^{7}$ Selden and Wei (2018) answer this question in the more general framework of KrepsPorteus preferences.

${ }^{8}$ Another detailed discussion, referring to this paper, is available in Gollier (2001), pp 146 and Proposition 75.
} 
equation provides clues to the solution to the differential inequality. For this case, the procedure looks like this: first, the differential equation counterpart to the local necessary condition, which gives the borderline case, is

$$
\frac{u^{\prime \prime \prime}(w)}{u^{\prime \prime}(w}=2 \frac{u^{\prime \prime}(w)}{u^{\prime}(w)}
$$

Assuming $u^{\prime}(w)$ is always positive and $u^{\prime \prime}(w)$ is always negative, this has the first integral

$$
\ln \left(-u^{\prime \prime}(w)\right)=2 \ln \left(u^{\prime}(w)\right)+\text { constant }
$$

or equivalently

$$
\frac{-u^{\prime \prime}(w)}{\left[u^{\prime}(w)\right]^{2}}=\text { constant }
$$

This in turn can be integrated to

$$
\frac{1}{u^{\prime}(w)}=a+b x
$$

That is, the borderline case is when the reciprocal of marginal utility is linear. To have decreasing marginal utility, $b$ must be positive. There are constraints on $a$ as well in order to keep marginal utility positive. Now, use the algebra for the differential equation case as a clue for solving the differential inequality. To keep signs straight, differential inequalities need to be solved by restatement with a combination of integration and differentiation, rather integrating as one would do for a differential equation. The differential inequality $P(w) \leq 2 A(w)$ can be written without the minus signs as

$$
\left.\frac{u^{\prime \prime \prime}(w)}{u^{\prime \prime}(w}\right) \geq 2 \frac{u^{\prime \prime}(w)}{u^{\prime}(w)}
$$

This differential inequality is equivalent to

$$
\frac{d}{d w} \ln \left(-u^{\prime \prime}(w)\right) \geq 2 \frac{d}{d w} \ln \left(u^{\prime}(w)\right)
$$

or

$$
\frac{d}{d w} \ln \left(\frac{-u^{\prime \prime}(w)}{\left[u^{\prime}(w)\right]^{2}}\right) \geq 0
$$


Because the natural logarithm is a monotonically increasing function, this in turn implies

$$
\frac{d}{d w}\left(\frac{-u^{\prime \prime}(w)}{\left[u^{\prime}(w)\right]^{2}}\right) \geq 0
$$

Finally, that condition is equivalent to

$$
\frac{d^{2}}{d w^{2}}\left(\frac{1}{u^{\prime}(w)}\right) \geq 0
$$

This is the condition of convexity of the reciprocal of marginal utility stated above.

For this problem, after "integrating" the differential inequality in this reinterpretive sense, the final step in checking whether the local $\mathrm{NC}$ holding true for all initial wealths is enough to guarantee the central NSC for any initial wealth is to rearrange the central NSC to show it is equivalent to the reciprocal of marginal utility being above the tangent lines to the reciprocal of marginal utility. That is, once one knows that the local necessary condition implies convexity of $1 / u^{\prime}(w)$, one can check whether any of the salient properties of convex functions help in establishing the truth of the central NSC.

One nice thing about this problem is that one can switch directions, and all the steps go through, with only a change in the direction of some of the inequalities. That is, if one asks "Under what condition does this new investment opportunity reduce consumption and increases saving today?" the answer is that guaranteeing this for any starting wealth and any risk pattern for the new investment opportunity requires that $1 / u^{\prime}(w)$ be concave. Demonstrating this is an excellent exercise for understanding the technique here.

It is possible for neither of these two conditions to hold: the reciprocal of marginal utility can be convex in some intervals and concave in others. But if one of them does hold globally, which is more likely? Consider constantrelative-risk-aversion utility functions with the functional form

$$
u(w)=\frac{w^{1-\gamma}-1}{1-\gamma}
$$

which limit into $\ln (w)$ for $\gamma=1$. Marginal utility is $u^{\prime}(w)=w^{-\gamma}$ and the reciprocal of marginal utility is

$$
\frac{1}{u^{\prime}(w)}=w^{\gamma} .
$$


This is convex if $\gamma>1$, concave if $\gamma<1$, and linear in the log-utility case $\gamma=1$. The value of $\gamma$ is controversial, so not everyone will agree, but based on the equity premium puzzle, $\gamma>1$ seems likely. Thus, assuming constant relatively risk aversion, it seems more likely that opening a new asset market will raise consumption and reduce saving. In terms of the inequality $P \leq 2 A$, in the special case of constant relative risk aversion $\gamma$, we have $A(w)=\gamma / w$ and $P(w)=(\gamma+1) / w$. This implies that $P \leq 2 A$ if and only if $\gamma \geq 1$.

To reprise the result:

Proposition 2 Opening up a new asset market delivering a net payoff in the future raises (reduces) current consumption if and only if absolute prudence is smaller (larger) than twice absolute risk aversion.

The condition that prudence is smaller (larger) than twice risk aversion is equivalent to the condition that risk tolerance $1 / A$ has a derivative that is smaller (larger) than one. These conditions are useful in many other comparative statics problems such as the impact of heterogeneous beliefs (Gollier (2007)) on asset prices. The fact that a logarithmic agent would not change current consumption when a new asset market with independent returns is opened is another illustration of the myopia of logarithmic agents (see for example Mossin (1968)).

\subsubsection{Effect of a new investment opportunity on the attitude to- ward another small risk}

Would opening up a new asset market induce risk-averse investors to reject a lottery that they would have accepted otherwise? Here we solve this problem for the case of small lotteries. In other words, we determine whether opening up a new market increases local risk aversion. If $\tilde{x}$ denotes the optimal exposure to the new risky investment opportunity at wealth level $w$, undertaking $\tilde{x}$ modifies the attitude towards other independent risks. In the small, this change in attitude is described by the change from the local concavity at $w$ of the original utility function $u$ to the local concavity at $w$ of the indirect utility function given by $v(z)=E u(z+\tilde{x})$. The problem can thus be written as follows:

$$
\forall \tilde{x}: \quad E \tilde{x} u^{\prime}(w+\tilde{x})=0 \quad \Longrightarrow \quad-\frac{E u^{\prime \prime}(w+\tilde{x})}{E u^{\prime}(w+\tilde{x})} \geq-\frac{u^{\prime \prime}(w)}{u^{\prime}(w)},
$$

After clearing fractions, applying the Diffidence Theorem yields the following condition:

$$
\forall x: A(w+x) \geq A(w)+x A^{\prime}(w) .
$$


The local necessary condition is $A^{\prime \prime}(w) \geq 0$. If we require that the individual becomes locally more risk-averse when a new investment opportunity appears, regardless of initial wealth level, then this local condition must hold for all $w$, i.e., absolute risk aversion must be convex. But this condition is obviously equivalent to Condition (45). This proves the following proposition, which is also in Gollier (2001, Proposition 33), referring to this paper.

Proposition 3 A new investment opportunity raises local risk aversion if and only if absolute risk aversion is convex.

The index of absolute risk aversion of all familiar utility functions is convex. In fact, all familiar utility functions have harmonic risk aversion, i.e., a linear risk tolerance.

\subsection{Applications with no univariate necessary and sufficient condition}

In this section, we present more difficult applications of the Diffidence Theorem in which the sufficient conditions in the small are not sufficient in the large.

\subsubsection{Effect of a unfair background risk on the equilibrium risk free rate}

Consider a simple Lucas tree model with two consumption dates. The crop from the tree is $c_{0}$ at date 0 , and $w$ at date 1 . The representative agent selects saving $s$ in order to maximize her discounted expected utility:

$$
\max _{s} v\left(c_{0}-s\right)+u_{i}\left(w+e^{r} s\right),
$$

where $r$ is the interest rate on savings. At equilibrium, assuming that $u_{i}$ is concave, we must have $s=0$, which yields the following classical pricing equation:

$$
e^{r_{i}}=\frac{v^{\prime}\left(c_{0}\right)}{u_{i}^{\prime}(w)}
$$

Suppose now that an unfair risk $\tilde{x}$ (i.e. $E \tilde{x} \leq 0$ ) is added to the fruit crop. It is easy to check that this reduces the equilibrium rate by $\Delta_{i}$, with

$$
\Delta_{i}=\log \left(\frac{E u_{i}^{\prime}(w+\tilde{x})}{u_{i}^{\prime}(w)}\right)
$$


$\Delta_{i}$ is usually referred to as the precautionary term, as it measures the downward sensitivity of the equilibrium interest rate to future risk. Under risk aversion and prudence, this precautionary term is positive. It plays an important role in the debate on the climate discount rate (Weitzman, 2007; Gollier, 2016), and in solving the risk-free rate puzzle. We are interested in characterizing the role of preferences on the size of this precautionary term. We compare two economies, $i=1,2$, that differ in the risk attitudes given by $u_{i}$. The effect of risk $\tilde{x}$ on the equilibrium interest rate is larger in economy 2 than in economy 1 iff

$$
\forall \tilde{x}: \quad E \tilde{x} \leq 0 \quad \Longrightarrow \quad \frac{E u_{2}^{\prime}(w+\tilde{x})}{u_{2}^{\prime}(w)} \geq \frac{E u_{1}^{\prime}(w+\tilde{x})}{u_{1}^{\prime}(w)},
$$

Thus, our problem simplifies to determining when does an unfair risk raise expected marginal utility by a bigger percentage for one utility function than another. Clearing fractions and then applying the Diffidence Theorem yields the following central NSC:

$$
\forall x: \frac{u_{1}^{\prime}(w+x)}{u_{1}^{\prime}(w)}-\frac{u_{2}^{\prime}(w+x)}{u_{2}^{\prime}(w)} \leq x\left[\frac{u_{1}^{\prime \prime}(w)}{u_{1}^{\prime}(w)}-\frac{u_{2}^{\prime \prime}(w)}{u_{2}^{\prime}(w)} .\right.
$$

The two local necessary conditions are

$$
\begin{aligned}
A_{2}(w) & \geq A_{1}(w) \\
A_{2}(w) P_{2}(w) & \geq A_{1}(w) P_{1}(w) .
\end{aligned}
$$

$A_{i}(w) P_{i}(w)=u_{i}^{\prime \prime \prime}(w) / u_{i}^{\prime}(w)$ is often referred to as the coefficient of downside risk aversion. ${ }^{9}$

This application of the Diffidence Theorem is our first example for which the local diffidence condition being true for all $w$ does not provide a global sufficient condition. That is, imposing Conditions (51) and (52) for all $w$ does not imply condition (50), which is what is needed to guarantee the comparative statics property (49) of interest.

But we hereafter show that the more restrictive condition $A_{2} \geq A_{1}$ and $P_{2} \geq P_{1} \geq 0$ is sufficient. Let $\psi_{i}$ denote the precautionary premium of risk $\tilde{x}$ for individual $i$, i.e., $E u_{i}^{\prime}(w+\tilde{x})=u_{i}^{\prime}\left(w-\psi_{i}\right)$. Because of prudence, we know that $\psi_{i}$ is positive. Because $P_{2}$ is larger than $P_{1}$, we also know that $\psi_{2}$ is larger than $\psi_{1}$. We then find that

$$
\frac{E u_{2}^{\prime}(w+\tilde{x})}{u_{2}^{\prime}(w)}=\frac{u_{2}^{\prime}\left(w-\psi_{2}\right)}{u_{2}^{\prime}(w)} \geq \frac{u_{1}^{\prime}\left(w-\psi_{2}\right)}{u_{1}^{\prime}(w)} \geq \frac{u_{1}^{\prime}\left(w-\psi_{1}\right)}{u_{1}^{\prime}(w)}=\frac{E u_{1}^{\prime}(w+\tilde{x})}{u_{1}^{\prime}(w)} .
$$

\footnotetext{
${ }^{9}$ Keenan and Snow (2010) discuss the different possible indices of downside risk aversion and their link to risk aversion and prudence.
} 
The first inequality comes from $\psi_{2} \geq 0$ and $A_{2} \geq A_{1}$. Indeed, this last condition implies that $u_{2}^{\prime}(w-x) / u_{2}^{\prime}(w)$ is larger than $u_{1}^{\prime}(w-x) / u_{1}^{\prime}(w)$ for all positive $x$. The second inequality in (53) comes from the fact that $\psi_{2}$ is larger than $\psi_{1}$ and $u_{1}$ is concave. This demonstrates the sufficiency of condition (i) of the following proposition. the proof of the sufficiency of part (ii) is relegated to the Appendix. ${ }^{10}$

Proposition 4 Introducing an unfair idiosyncratic risk to consumption growth has a larger effect on the equilibrium risk-free rate in the economy with utility function $u_{2}$ than in the economy with utility function $u_{1}$ only if $A_{2} \geq A_{1}$ and $A_{2} P_{2} \geq A_{1} P_{1}$. The following two conditions are each sufficient:

(i) $A_{2} \geq A_{1}$ and $P_{2} \geq P_{1} \geq 0$;

(ii) $u_{2}^{\prime \prime}-\frac{u_{2}^{\prime}}{u_{1}^{\prime}} u_{1}^{\prime \prime}$ is increasing.

Using the Diffidence Theorem, Gollier (2015) addressed the associated question of the impact of wealth inequality on the equilibrium interest rate. In particular, he shows that the wealth inequality increases the interest rate if relative risk aversion is decreasing and convex.

\subsubsection{Acceptance of a portfolio}

Consider again the one-safe-one-risky-asset portfolio problem. Is the portfolio selected by individual 1 desirable for individual 2, when individual 2 only has a binary choice between that portfolio and the fully risk-free option? This problem might be encountered by a fund manager or a life insurer who wishes to select a portfolio that maximizes the welfare of the modal customer, while at the same time keeping more risk-averse individuals invested in the fund. Individual 2 finds individual 1's optimal quantity of the risky asset acceptable compared to none iff:

$$
\forall \tilde{x}: \quad E \tilde{x} u_{1}^{\prime}(w+\tilde{x})=0 \quad \Longrightarrow \quad E u_{2}(w+\tilde{x}) \geq u_{2}(w),
$$

Applying the Diffidence Theorem yields the following central NSC:

$$
\forall x: u_{2}(w+x)-u_{2}(w) \geq \frac{u_{2}^{\prime}(w)}{u_{1}^{\prime}(w)} x u_{1}^{\prime}(w+x)
$$

The local necessary condition that bites is:

$$
A_{2}(w) \leq 2 A_{1}(w) .
$$

\footnotetext{
${ }^{10}$ The results presented in Proposition 4 have not been published elsewhere.
} 
We obtain the following Proposition.

Proposition $5 A$ necessary condition for $u_{2}$ to like the optimal portfolio of $u_{1}$ is $A_{2} \leq 2 A_{1}$. A sufficient condition is $A_{2} \leq P_{1} \leq 2 A_{1}$.

Proof: Sufficiency of $A_{2} \leq P_{1} \leq 2 A_{1}$ comes from the fact that the second inequality implies $\left[E \tilde{x} u_{1}^{\prime}(w+\tilde{x})=0 \Longrightarrow E u_{1}^{\prime}(w+\tilde{x}) \leq u_{1}^{\prime}(w)\right]$, while the first inequality implies that $\left[E u_{1}^{\prime}(w+\tilde{x}) \leq u_{1}^{\prime}(w) \Longrightarrow E u_{1}(w+\tilde{x}) \geq u_{1}(w)\right]$.

Remark: Recall that $P_{1} \leq 2 A_{1}$ is satisfied for CRRA if $\gamma>1$. Also recall that $P=\gamma+1$ for CRRA. Hence, if the modal investor has constant relative risk aversion larger than 1 , the amount of the risky asset chosen to optimize for the modal customer is guaranteed to be acceptable to others who have constant relative risk aversion $\gamma_{2}$ with $\gamma_{2} \leq \gamma_{1}+1$.

Symmetrically, agent $u_{2}$ always dislikes the optimal portfolio of agent $u_{1}$ only if $A_{2} \geq 2 A_{1}$. A sufficient condition is $A_{2} \geq P_{1} \geq 2 A_{1}$.

\subsubsection{Risk vulnerability}

Gollier and Pratt (1996) defined the concept of "risk vulnerability" as follows: $u_{1}$ is vulnerable to risk if adding an unfair background risk to wealth raises the aversion to any other independent risk, namely:

$$
\forall \tilde{x}: \quad E \tilde{x} \leq 0 \quad \Longrightarrow \quad-\frac{E u_{1}^{\prime \prime}(w+\tilde{x})}{E u_{1}^{\prime}(w+\tilde{x})} \geq-\frac{u_{1}^{\prime \prime}(w)}{u_{1}^{\prime}(w)},
$$

Equivalently, assuming $u^{\prime \prime}(w)<0$,

$$
\forall \tilde{x}: \quad E \tilde{x} \leq 0 \quad \Longrightarrow \quad \frac{E u_{1}^{\prime \prime}(w+\tilde{x})}{u_{1}^{\prime \prime}(w)} \geq \frac{E u_{1}^{\prime}(w+\tilde{x})}{u_{1}^{\prime}(w)},
$$

Let $T_{i}(w)=-u_{i}^{\prime \prime \prime \prime}(w) / u_{i}^{\prime \prime \prime}(w)$ denote the index of absolute temperance as defined in Gollier and Pratt (1996). When comparing problem (58) to problem (49), we see that risk vulnerability is an application of problem (49) by defining $u_{2} \equiv-u_{1}^{\prime}$. Therefore, after some algebra, the central NSC is:

$$
u_{1}^{\prime}(w+x)\left[A_{1}(w+x)-A_{1}(w)\right] \geq x u_{1}^{\prime}(w) A_{1}^{\prime}(w),
$$

the necessary conditions $P_{1} \geq A_{1}$ and $T_{1} \geq A_{1}$, together with the sufficient condition $T_{1} \geq P_{1} \geq A_{1}$ obtained by Gollier and Pratt (1996) are directly obtained from Conditions (50), (51), (52) and $\left(P_{2} \geq P_{1}, A_{2} \geq A_{1}\right)$. 
The role of background risk in optimal risk management has been extensively studied over the last two decades by using the Diffidence Theorem. For example, Crainich, Eeckhoudt and Le Courtois (2016) extend the analysis to the case of bivariate utility functions. Making the second argument $y$ (health, for example) of the utility function risky lowers the propensity to take financial risk only if the intensity of cross prudence defined by $u_{x y y} / u_{x}$ decreases with wealth $x .{ }^{11}$ Franke, Schlesinger and Stapleton (2011) characterize the concept corresponding to risk vulnerability when the background risk is multiplicative. Hara, Huang and Kuzmics (2011) characterize the impact of an independent additive background risk on the demand for portfolio insurance. Franke, Stapleton and Subrahmanyam (1998) characterize the impact of background risk on the demand for portfolio insurance when absolute risk tolerance is linear in wealth within a general equilibrium framework. Eichner [2008], Jiang, Ma and An (2010) and Eichner and Wagener (2012) examine the optimal composition of the assets portfolio in the presence of background risk in the mean-variance context. Testlin and Winkler (2005) are the first to generalize the concept of risk vulnerability to the case of correlated background risks. Arrondel, Calvo-Pardo and Oliver (2010) estimate the degree of risk vulnerability and temperance by using French cross-sectional data on earnings and portfolio choices. Finally, Gollier and Schlesinger (2002) discuss the implications of background risk and risk vulnerability for the equity premium.

\subsubsection{Semi properness}

Pratt and Zeckhauser (1987) introduce the notion of properness, which guarantees that adding any undesirable background risk to wealth makes the agent more averse to any other independent risk. In this subsection, we introduce the notion of semi-properness. Utility function $u$ is semi-proper if adding any undesirable background risk to wealth makes the agent more averse to any other small independent risk. This means that the indirect utility function $v(w)=E u(w+\tilde{x})$ is locally more concave than $u$ at all wealth levels at which $\tilde{x}$ is undesirable:

$$
\forall \tilde{x}: \quad E u(w+\tilde{x}) \leq u(w) \quad \Longrightarrow \quad-\frac{E u^{\prime \prime}(w+\tilde{x})}{E u^{\prime}(w+\tilde{x})} \geq-\frac{u^{\prime \prime}(w)}{u^{\prime}(w)}
$$

As it is apparent, semi properness is a weaker restriction than properness. Indeed, semi properness means that indirect utility function $v$ is required

\footnotetext{
${ }^{11}$ For more on cross-prudence, see Eeckhoudt, Rey and Schlesinger (2007).
} 
to be locally more risk-averse than $u$ at $w$. This is weaker than properness, which requires that $v$ be centrally more diffident than $u$ at $w$, a condition that is stronger than locally greater risk aversion as shown in subsection 3.1.2.

However, semi-properness is a stronger restriction than risk vulnerability. This is apparent from the fact that the same condition $A_{v}(w) \geq A_{1}(w)$ is required to hold for a larger set of random variables under semi properness than under risk vulnerability. Indeed, under risk aversion, the set of undesirable lotteries contains the set of non-positive-mean lotteries as a subset.

Clearing fraction and then using the Diffidence Theorem, the necessary and sufficient condition for semi properness can be written as follows:

$$
\forall x: \frac{A(w+x)-A(w)}{A^{\prime}(w)} \leq \frac{u(w+x)-u(w)}{u^{\prime}(w+x)}
$$

The local necessary conditions are $A^{\prime}(w) \leq 0$ and $A^{\prime \prime}(w) \geq A^{\prime}(w) A(w)$. These conditions, even when imposed for all wealth levels $w$, are not sufficient for (61). But suppose that $A^{\prime} \leq 0$ and that $-A$ is more concave than $u$, i.e., that $-A^{\prime \prime} / A^{\prime}$ is uniformly larger than $A$. Using our findings in subsection 3.1.2, this implies the first inequality below:

$$
\frac{A(w+x)-A(w)}{A^{\prime}(w)} \leq \frac{u(w+x)-u(w)}{u^{\prime}(w)} \leq \frac{u(w+x)-u(w)}{u^{\prime}(w+x)},
$$

for all $x$. The second inequality is a consequence of the concavity of $u$. This yields the sufficient condition (61) and thereby demonstrates the following proposition.

Proposition 6 Adding an undesirable risk to wealth raises the aversion to any other small risk only if $A^{\prime} \leq 0$ and $A^{\prime \prime} \geq A^{\prime} A$. A sufficient condition is $A^{\prime} \leq 0$ and $A^{\prime \prime} \geq-A^{\prime} A$.

Remark: For CRRA, $-A(w)=-\gamma / w$, so $-A^{\prime \prime}(w) / A^{\prime}(w)=2 / w$. This means that for the CRRA case, the sufficient condition in the proposition above applies when relative risk aversion is $\leq 2$.

Rather than examining the impact of the introduction of risk on the attitude towards other independent risks, Eeckhoudt, Gollier and Schlesinger (1996) and Keenan, Rudow and Snow (2008) explore the impact of an nth-degree stochastic deterioration of a background risk. Lajeri-Chaherli 
(2002) examine properness in the mean-variance framework. Lajeri-Chaherli showed that quasi-concavity plus decreasing risk aversion is equivalent to proper risk aversion in that context. In Lajeri-Chaherli (2004), they extend these ideas to two-moment decision models. Munier and Tapiero (2008) explore the generalization of properness and similar concepts to non-expectedutility models.

\section{The Bivariate Diffidence Theorem}

Other problems involving multiple risks require a modification of the techniques of the Diffidence Theorem. Pratt and Zeckhauser (1987) define proper risk aversion by requiring that any undesirable risk can never be made desirable by adding another independent undesirable risk to wealth. ${ }^{12}$ Mathematically, (fixed-wealth) properness is defined by the following property: $\forall w, \tilde{x}, \tilde{y}$ :

$E u(w+\tilde{x}) \leq u(w)$ and $E u(w+\tilde{y}) \leq u(w) \Longrightarrow E u(w+\tilde{y}+\tilde{x}) \leq E u(w+\tilde{x})$,

where $\tilde{x}$ and $\tilde{y}$ are independent random variables. There is a simple way to solve this problem by using the Diffidence Theorem twice. Indeed, by definition, $u_{1}$ is proper if and only if $v(w)=E u(w+\tilde{x})$ is centrally more risk-averse than $u$ for any undesirable risk $\tilde{x}$ at $w$, and for any $w$. Thus, an equivalent definition of properness is: $\forall w, y, \tilde{x}: E u(w+\tilde{x}) \leq u(w)$ implies

$$
\frac{E u(w+y+\tilde{x})-E u(w+\tilde{x})}{E u^{\prime}(w+\tilde{x})} \leq \frac{u(w+y)-u(w)}{u^{\prime}(w)} .
$$

Applying the Diffidence Theorem again for every $y$ yields the necessary and sufficient condition obtained by Pratt and Zeckhauser (1987, Theorem 4).

Taking inspiration from this example, let us state and prove the Bivariate Diffidence Theorem. The general structure of the problem that can be solved by the Bivariate Diffidence Theorem is as follows: ${ }^{13}$

$$
\forall \tilde{x}, \tilde{y}: \quad E f_{1}(\tilde{x})=f_{1}(0) \text { and } E f_{2}(\tilde{y})=f_{2}(0) \quad \Longrightarrow \quad E h(\tilde{x}, \tilde{y}) \leq 0 .
$$

\footnotetext{
${ }^{12}$ More recently, the bivariate Diffidence Theorem has been used by Crainich, Eeckhoudt and Le Courtois (2014) to link risk vulnerability to decreasing downside risk aversion.

${ }^{13}$ To be concise, we limit the analysis to nonstochastic initial $w$, and equality conditions. Other formulations can easily be characterized by following the same rules as above.
} 
In the following bivariate Diffidence Theorem, we systematize the technique that has been presented above to characterize properness.

Theorem 2 Suppose that function $h(x, y)$ satisfies $h(x, 0) \equiv 0$ and $h(0, y) \equiv$ 0 . Then, as long as $f_{1}^{\prime}(0) \neq 0$ and $f_{2}^{\prime}(0) \neq 0$, Condition (64) is equivalent to the condition that

$$
h(x, y)-\delta_{1}(x) \frac{\partial h}{\partial x}(0, y)-\delta_{2}(y) \frac{\partial h}{\partial y}(x, 0)+\delta_{1}(x) \delta_{2}(y) \frac{\partial^{2} h}{\partial x \partial y}(0,0) \leq 0,
$$

for all $x$ and $y$, with $\delta_{i}(x)=\left(f_{i}(x)-f_{i}(0)\right) / f_{i}^{\prime}(0)$.

Proof: Given the assumptions, for any $\tilde{y}$ satisfying the second condition in (64), any $\tilde{x}$ satisfying the first condition in (64) implies $E h(\tilde{x}, \tilde{y}) \leq 0$. Consider any random variable $\tilde{y}$ such that $E f_{2}(\tilde{y})=f_{2}(0)$. Then, for this $\tilde{y}$, condition (64) is equivalent to the generic diffidence property (1). Then, the univariate Diffidence Theorem can be applied. It yields the following necessary and sufficient condition: For all $x$,

$$
E_{y}\left[h(x, \tilde{y})-\delta_{1}(x) \frac{\partial h}{\partial x}(0, \tilde{y})\right] \leq 0
$$

with $\delta_{1}(x)=\left(f_{1}(x)-f_{1}(0)\right) / f_{1}^{\prime}(0)$. This condition must hold for all $\tilde{y}$ such that $E f_{2}(\tilde{y})=f_{2}(0)$. The univariate Diffidence Theorem can be applied again to obtain (65) as a necessary and sufficient condition for (64).

Note: $h(x, y)$ satisfying $h(x, 0) \equiv 0$ and $h(0, y) \equiv 0$ is equivalent to:

$$
h(x, y)=j(x, y)-j(x, 0)-j(0, y)+j(0,0)
$$

for some function $j(x, y)$. It is clear that for any $j$ this gives an $h$ with the stipulated property. On the other hand, if one has $h$ in hand, it is easy to find a $j$ - just take $h$ itself as $j$.

There are many other applications of the bivariate diffidence theorem. For example, if the first condition in (62) is replaced by $E u^{\prime}(w+\tilde{x}) \geq u^{\prime}(w)$, we get the notion of standard risk aversion introduced by Kimball (1993). If it is replaced by condition $E \tilde{x} \leq 0$, we get risk vulnerability. It would also be easy to apply the Theorem to the following problem: $\forall w, \tilde{x}, \tilde{y}$ :

$E \tilde{x} u_{1}^{\prime}(w+\tilde{x})=0$ and $E u_{1}(w+\tilde{y})=u_{1}(w) \Rightarrow E u_{1}(w+\tilde{y}+\tilde{x}) \leq E u_{1}(w+\tilde{x})$. 
This is the problem of guaranteeing that opening up a new asset market makes undesirable any risk for which one was indifferent prior to the opening. Obviously, this condition is stronger than (44). This is left as an exercise for interested readers. Many other examples can be found by combining different types of functions $f_{i}$ that we considered in the previous section.

\section{Other extensions}

\subsection{Allowing for an arbitrary reference situation}

All applications of the Diffidence Theorem above rely on a pre-specified initial wealth level $w$. But one can imagine applications in which the initial distribution of wealth is not specified. What would happen to the characterization of the problem if the distribution of initial wealth $\tilde{\omega}$ is stochastic and arbitrary? In other words, what are the restrictions on functions $f_{1}, f_{2}$ to get

$$
\forall \tilde{x}, \tilde{\omega}: \quad E f_{1}(\tilde{x}) \leq E f_{1}(\tilde{\omega}) \Longrightarrow E f_{2}(\tilde{x}) \leq E f_{2}(\tilde{\omega}),
$$

To illustrate, if these functions are utility functions, under what conditions on preferences is it true that if agent $u_{1}$ prefers a lottery to another, then agent $u_{2}$ also prefers the former to the latter? We know the answer to this simple problem: the two functions must represent the same preferences, i.e. $\exists m>0: f_{2}(.) \equiv m f_{1}()+$.$b . We generalize this result in the following$ Proposition, which can be easily demonstrated using the Diffidence Theorem.

Proposition 7 Condition (68) holds if and only if there exists a positive scalar $m$ such that $f_{2}(x)-f_{2}(0)=m\left[f_{1}(x)-f_{1}(0)\right]$.

\subsection{The Multi-Antecedent Diffidence Theorem}

Another possible extension is to consider more than one condition on $\tilde{x}$. Namely, consider an index set $\Theta \subset R$ that can be either finite or infinite, and a set of functions $\left\{f_{\theta}(x), \theta \in \Theta\right\}$. The multi-antecedent problem is written as:

$$
\forall \tilde{x}: \quad E f_{\theta}(\tilde{x}) \leq E f_{\theta}\left(\tilde{\omega}_{\theta}\right) \forall \theta \in \Theta \Longrightarrow E g(\tilde{x}) \leq E g(\tilde{\omega}),
$$

where $\left\{\tilde{\omega}_{\theta} \mid \theta \in \Theta\right\}$ is a prespecified set of random variables. The multiantecedent Diffidence Theorem provides a tool to solve this kind of problem. 
Theorem 3 Suppose that functions $f_{\theta}$ and $g$ are in $L_{2}[a, b]$. Then Condition (69) holds if and only if there exists a nonnegative Lebesgues-integrable function $m: \Theta \rightarrow R$ such that

$$
g(x)-E g(\tilde{\omega}) \leq \int_{\Theta} m(\theta)\left[f_{\theta}(x)-E f_{\theta}\left(\tilde{\omega}_{\theta}\right)\right] d \theta,
$$

for all $x$ in $[a, b]$.

Proof: See the Appendix.

Notice that multiplying the right-hand-side of Condition (70) by an additional nonnegative scalar does not change the substance of the condition. Thus, condition (69) is equivalent to the property

$$
\forall \tilde{x}: \quad E \int_{\Theta} m(\theta) f_{\theta}(\tilde{x}) d \theta \leq E \int_{\Theta} m(\theta) f_{\theta}(\tilde{\omega}) d \theta \Longrightarrow E g(\tilde{x}) \leq E g(\tilde{\omega}) .
$$

This means that any problem with more than one antecedent is equivalent to another problem with only one antecedent on which the single-antecedent Diffidence Theorem can be applied. To illustrate, consider the problem of an agent $v$ rejecting any lottery that both agents $u_{1}$ and $u_{2}$ reject:

$$
\forall \tilde{x}: \quad E u_{i}(w+\tilde{x}) \leq u_{i}(w), i=1,2 \Longrightarrow E v(w+\tilde{x}) \leq v(w) .
$$

From our results, this is possible only if there exists two nonnegative scalars $m_{1}, m_{2}$ such that $v$ is centrally more risk-averse around $w$ than $u_{3}=m_{1} u_{1}+$ $m_{2} u_{2}$.

\subsection{The Multi-Antecedent Multi-Reference Diffidence The- orem}

Of course, one can easily combine the two previous results to analyze problems with more than one antecedent and with an arbitrary reference point:

$$
\forall \tilde{x}, \tilde{\omega}: \quad E f_{\theta}(\tilde{x}) \leq E f_{\theta}\left(\tilde{\omega}_{\theta}\right) \forall \theta \in \Theta \Longrightarrow E g(\tilde{x}) \leq E g(\tilde{\omega}) .
$$

This is possible only if there exists a nonnegative function $m: \Theta \rightarrow R$ such that

$$
g(x)=\int_{\Theta} m(\theta) f_{\theta}(x) d \theta .
$$


Jewitt (1987) obtains this condition. He applied it to the Ross' concept of "strongly greater risk aversion" (Ross, 1981). A simpler illustration is to determine under what condition agent $v$ dislikes any change in risk that agents both $u_{1}$ and $u_{2}$ dislike. From the discussion above, this is possible if and only if $v$ is a convex combination of $u_{1}$ and $u_{2}$. More generally, if an agent $v$ dislikes any change in risk that any agent with constant absolute risk aversion dislikes, it must be the case that $v$ is completely monotone, i.e. all odd derivatives of $v$ must be positive and all even derivatives of $v$ must be negative: $v(w)=-\int_{0}^{+\infty} m(\theta) e^{-\theta w} d \theta$.

\section{Concluding remarks}

Under what condition on $\left(f_{1}, f_{2}\right)$ do we have the property that $E f_{2}(w, \tilde{x}) \leq$ $f_{2}(w, 0)$ for all lotteries $\tilde{x}$ for which $E f_{1}(w, \tilde{x})=f_{1}(w, 0)$ ? The Diffidence Theorem points to three different conditions associated to this problem. The simplest one consists in a necessary condition that is in fact necessary and sufficient "in the small", i.e., when we limit the set of random variables to small risks. This necessary condition states that $f_{2}(w, x)$ must be more concave with respect to $x$ in the sense of Arrow-Pratt than $f_{1}(w, x)$, locally in the neighborhood of $(w, 0)$. This local condition is generally straightforward to characterize by standard univariate calculus.

The second condition is called a "central" condition, and is necessary and sufficient for the property above to hold for all $\tilde{x}$, but for a fixed, given $w$. Finally, the "global" condition requires that the property holds for all $w$. The Diffidence Theorem fully characterizes the central and global conditions, the latter requiring that a bivariate function be nonpositive. Of course, the global condition implies the central condition, which in turn implies the local one, but the reverse is not true in general. However, there are circumstances, such as when functions $f_{1}$ and $f_{2}$ are additive in $(w, x)$, in which the local condition implies the global one. But in many applications, such as those associated to the concepts of properness and risk vulnerability, the condition "in the small" is not equivalent to the condition "in the large". This paper describes these local necessary conditions, central necessary and sufficient conditions and global sufficient conditions, and discusses their links in the context of a wide variety of applied problems. One key unresolved question is: What general principle determines when the local condition holding for all initial $w$ implies the global condition? 


\section{References}

Arrondel, L., H. Calvo Pardo, and X. Oliver, (2010), Temperance in stock market participation: Evidence from France, Economica 77, 314-333.

Crainich, D., L. Eeckhoudt, and O. Le Courtois, (2014), Decreasing downside risk aversion and background risk, Journal of Mathematical Economics 53, 59-63.

Crainich, D., L. Eeckhoudt, and O. Le Courtois, (2016), Health and portfolio choices: A diffidence approach, European Journal of Operational Research 259, 273-279.

Dionne, G., and M.-G. Ingabire, (2001), Diffidence theorem, state dependent preferences, and DARA, The Geneva Papers on Risk and Insurance Theory, 26, 139-154.

Doherty, N.A., and H. Schlesinger, (1983), Optimal insurance in incomplete markets, Journal of Political Economy 91, 10451054 .

Eeckhoudt, L., C. Gollier, and H. Schlesinger, (1996), Changes in Background Risk, and Risk Taking Behaviour, Econometrica, 64, 683-690.

Eeckhoudt, L., C. Gollier, and T. Schneider, (1995), Risk Aversion, Prudence and Temperance: A Unified Approach, Economics Letters, 48, 331-336.

Eeckhoudt, L., B. Rey, and H. Schlesinger, (2007), A Good Sign for Multivariate Risk Taking, Management Science, 53, 117124 .

Eeckhoudt, L., and H. Schlesinger, (2006), Putting risk in its proper place, American Economic Review 96, 280-289.

Eichner, T., (2008), Mean variance vulnerability, Management Science 54, 586-593.

Eichner, T., and A. Wagener, (2012), Tempering effects of (dependent) background risks: A mean-variance analysis of portfolio selection, Journal of Mathematical Economics 48, 422430 .

Franke, G., H. Schlesinger, and R.C. Stapleton, (2011), Risk taking with additive and multiplicative background risks, Journal of Economic Theory 146, 1147-1568. 
Franke, G., R.C. Stapleton, and M.G. Subrahmanyam, (1998), Who Buys and Who Sells Options: The Role of Options in an Economy with Background Risk, Journal of Economic Theory 82, 89-109.

Gollier, C., (1996), Decreasing Absolute Prudence: Characterization and Applications to Second-Best Risk Sharing, European Economic Review 40, 1799-1815.

Gollier, C., (2001), The economics of risk and time, MIT Press.

Gollier, C., (2007), Whom should we believe? Aggregation of heterogeneous beliefs, Journal of Risk and Uncertainty 35, 107-127.

Gollier, C., (2015), Discounting, inequality and economic convergence, Journal of Environmental Economics and Management 69, 53-61.

Gollier, C., (2016), Evaluation of long-dated assets : The role of parameter uncertainty, Journal of Monetary Economics 84, 66-83.

Gollier, C. and M.S. Kimball, (1994), Toward a systematic approach to the economic effects of risk II: Characterizing utility functions, unpublished manuscript, University of Toulouse.

Gollier, C. and M.S. Kimball, (2018), New Methods in the Classical Economics of Uncertainty : Comparing Risks, Geneva Risk and Insurance Review, forthcoming.

Gollier, C., and J.W. Pratt, (1996), Risk Vulnerability and the Tempering Effect of Background Risk, Econometrica 64, 1109-1124. Gollier, C., and H. Schlesinger, (2002), Changes in risk and asset prices, Journal of Monetary Economics 49, 761-795.

Hara, C., J. Huang, and C. Kuzmics, (2011), Effects of background risks on cautiousness with an application to a portfolio choice problem, Journal of Economic Theory 146, 346358.

Jewitt, I., (1987), A Note on comparative statics and stochastic dominance, Journal of Mathematical Economics 15, 259-254.

Jiang, C., Y. Ma, and Y. An, (2010), An analysis of portfolio selection with background risk, Journal of Banking and Finance 34, 3055-3060. 
Lajeri-Chaherli, F., (2002), More on Properness: The Case of Mean-Variance Preferences, The Geneva Papers on Risk and Insurance Theory 27, 49-60.

Lajeri-Chaherli, F., (2004), Proper and standard risk aversion in two-moment decision models. Theory and Decision 57, 213-225.

Kihlstrom, R., D. Romer and S. Williams, (1981), Risk Aversion with Random Initial Wealth, Econometrica49, 911-920.

Keenan, D., D.C. Rudow, and A. Snow, (2008), Risk preferences and changes in background risk, Journal of Risk and Uncertainty 36, 139-152.

Keenan, D., and A. Snow, (2010), Greater prudence and greater downside risk aversion, Journal of Economic Theory 145, 2018-2026.

Kimball, M.S., (1990), Precautionary Saving in the Small and in the Large, Econometrica 58, 53-73.

Kimball, M.S., (1993), Standard Risk Aversion, Econometrica $61,589-611$.

Mossin, J., (1968), Optimal Multiperiod Portfolio Policies, Journal of Business 41, 215-229.

Munier, B. R., and C. Tapiero, (2008), Risk Attitude, in Encyclopedia of Quantitative Risk Analysis and Assessment IV, Wiley.

Pratt, J.W., (1964), Risk Aversion in the Small and in the Large, Econometrica, 32, 122-136.

Pratt, J.W. and R. Zeckhauser, (1987), Proper Risk Aversion, Econometrica 55, 143-154.

Ross, S.A., (1981), Some stronger measures of risk aversion in the small and in the large with applications, Econometrica 49, 621-638.

Selden, L, and X. Wei, (2018), Capital risk: Precautionary and excess saving, mimeo, Columbia University.

Testlin, I., and R. L. Winkler, (2005), Risky Choices and Correlated Background Risk, Management Science 51, 13336-1345.

Weil, P., (1989), The Equity Premium Puzzle and the Riskfree Rate Puzzle, Journal of Monetary Economics, 24, 401-421. 
Weil, P., (1992), Equilibrium Asset Prices with Undiversifiable Labor Income Risk, Journal of Economic Dynamics and Control 16, 769-790.

Weitzman, M. L., (2007), Subjective expectations and assetreturn puzzle, American Economic Review 97, 1102-1130. 


\section{APPENDIX}

\section{Proof of Proposition 4 (sufficiency of condition (ii))}

The two necessary conditions are simple rewritings of conditions (51)

and (52). The sufficiency of $u_{2}^{\prime \prime}(w)-\frac{u_{2}^{\prime}(w)}{u_{1}^{\prime}(w)} u_{1}^{\prime \prime}(w)$ increasing is obtained in the following way. Take any positive scalar $\xi$. The above condition means that

$$
u_{2}^{\prime \prime}(w+\xi)-\frac{u_{2}^{\prime}(w+\xi)}{u_{1}^{\prime}(w+\xi)} u_{1}^{\prime \prime}(w+\xi)-u_{2}^{\prime \prime}(w)+\frac{u_{2}^{\prime}(w)}{u_{1}^{\prime}(w)} u_{1}^{\prime \prime}(w) \geq 0 .
$$

Dividing by $u_{2}^{\prime}(w)$ and integrating the corresponding condition on $[0, x]$, $x>0$, yields

$$
\int_{0}^{x} \frac{u_{2}^{\prime \prime}(w+\xi)}{u_{2}^{\prime}(w)}-\frac{u_{1}^{\prime \prime}(w+\xi)}{u_{1}^{\prime}(w)} \frac{\frac{u_{2}^{\prime}(w+\xi)}{u_{2}^{\prime}(w)}}{\frac{u_{1}^{\prime}(w+\xi)}{u_{1}^{\prime}(w)}} d \xi \geq x\left[\frac{u_{2}^{\prime \prime}(w)}{u_{2}^{\prime}(w)}-\frac{u_{1}^{\prime \prime}(w)}{u_{1}^{\prime}(w)}\right]
$$

The same inequality holds for $x<0$ since the change of the direction of inequality (75) is compensated by the direction of the integration. So, for any $x$, we obtain

$$
\int_{0}^{x} \frac{u_{2}^{\prime \prime}(w+\xi)}{u_{2}^{\prime}(w)}-\frac{u_{1}^{\prime \prime}(w+\xi)}{u_{1}^{\prime}(w)} d \xi+g(x) \geq x\left[\frac{u_{2}^{\prime \prime}(w)}{u_{2}^{\prime}(w)}-\frac{u_{1}^{\prime \prime}(w)}{u_{1}^{\prime}(w)}\right]
$$

or, equivalently,

$$
\left[\frac{u_{2}^{\prime}(w+x)}{u_{2}^{\prime}(w)}-\frac{u_{1}^{\prime}(w+x)}{u_{1}^{\prime}(w)}\right]+g(x) \geq x\left[\frac{u_{2}^{\prime \prime}(w)}{u_{2}^{\prime}(w)}-\frac{u_{1}^{\prime \prime}(w)}{u_{1}^{\prime}(w)}\right]
$$

where

$$
g(x)=\int_{0}^{x} \frac{u_{1}^{\prime \prime}(w+\xi)}{u_{1}^{\prime}(w)}\left[\frac{\frac{u_{1}^{\prime}(w+\xi)}{u_{1}^{\prime}(w)}-\frac{u_{2}^{\prime}(w+\xi)}{u_{2}^{\prime}(w)}}{\frac{u_{1}^{\prime}(w+\xi)}{u_{1}^{\prime}(w)}}\right] d \xi .
$$

Because $u_{2}$ is more risk-averse than $u_{1}, u_{2}$ is centrally more risk-averse than $u_{1}$ around $w$. It implies that the bracketed term in the integrant of $g$ has the same sign as $x$. It implies that $g(x)$ is always negative. Combining this fact with condition (76) yields the sufficient condition (50).

\section{Proof of Theorem 3}


Consider the set of Lebesgue-integrable real functions in $L_{2}[a, b]$, with the associated product scalar $\langle f, g\rangle=\int f g$. If $h$ is a density function for $\tilde{x}$, then, $\left\langle f, h>\right.$ represents $E f(\tilde{x})$. Consider any subset $D \subset L_{2}[a, b]$ and define :

- $\hat{D}=\left\{G \in L_{2} \mid \forall h \in L_{2} \quad: \quad<G, h>\leq 0\right.$ whenever $<F, h>\leq 0 \quad \forall f \in$ $D\}$

- $C(D)=$ the smallest closed convex cone containing $D$.

To prove Theorem 3, we need first to prove the following Lemma, which is also used by Jewitt (1987).

Lemma : $\hat{D}=C(D)$

Proof: The fact that $C(D) \subset \hat{D}$ is obvious. Let us then prove $\hat{D} \subset C(D)$. By contradiction, suppose that $\exists \bar{G} \in \hat{D}$, but $\bar{G} \notin C(D)$. Since $C(D)$ is convex, one may apply a separation theorem : $\exists m \in L_{2}[a, b]:\langle\bar{G}, m\rangle \geq 0$ and $\langle F, m>\leq 0 \forall F \in C(D)$. It implies that $\bar{G} \notin \hat{D}$, a contradiction.

We are now in a position to prove Theorem 3. Take $D=\left\{F_{\theta}=f_{\theta}-\right.$ $\left.E f_{\theta}\left(\tilde{\omega}_{\theta}\right) \mid \theta \in \Theta\right\} \cup D_{-}$, with $D_{-}=\{k \mid k(x) \leq 0 \quad \forall x\}$, the set of non positive functions. Thus,

$$
\begin{gathered}
\hat{D}=\left\{G \mid \forall h \in L_{2}:<G, h>\leq 0\right. \text { whenever } \\
\left.<F_{\theta}, h>\leq 0 \forall \theta \in \Theta \text { and }<k, h>\leq 0 \quad \forall k \in D_{-}\right\}
\end{gathered}
$$

We introduced the set of conditions $\left\langle k, h>\leq 0 \quad \forall k \in D_{-}\right.$to ensure that $h$ may be interpreted as a density function. Thus, one can interpret $\hat{D}$ as

$$
\hat{D}=\left\{G \mid E G(\tilde{x}) \leq 0 \text { whenever } E F_{\theta}(\tilde{x}) \leq 0 \forall \theta \in \Theta\right\}
$$

Applying the lemma yields that $G \in \hat{D}$ if and only if there exist a function $k \in D_{-}$and a nonnegative function $m$, such that

$$
G(x) \leq \int_{\Theta} m(\theta) F_{\theta}(x) d \theta+k(x)
$$

for all $x$. Define $G=g-E g(\tilde{\omega})$. The above condition is thus equivalent to

$$
g(x)-E g(\tilde{\omega}) \leq \int_{\Theta} m(\theta)\left[f_{\theta}(x)-E f_{\theta}\left(\tilde{\omega}_{\theta}\right)\right] d \theta+k(x)
$$

for all $x$. This is equivalent to $(70)$, since $k(x) \leq 0$. 